\title{
Capacity of Large-scale CSMA Wireless Networks
}

\author{
Chi-Kin Chau, Minghua Chen, and Soung Chang Liew
}

\begin{abstract}
In the literature, asymptotic studies of multi-hop wireless network capacity often consider only centralized and deterministic TDMA (time-division multi-access) coordination schemes. There have been fewer studies of the asymptotic capacity of large-scale wireless networks based on CSMA (carriersensing multi-access), which schedules transmissions in a distributed and random manner. With the rapid and widespread adoption of CSMA technology, a critical question is that whether CSMA networks can be as scalable as TDMA networks. To answer this question and explore the capacity of CSMA networks, we first formulate the models of CSMA protocols to take into account the unique CSMA characteristics, not captured by existing interference models in the literature. These CSMA models determine the feasible states, and consequently the capacity of CSMA networks. We then study the throughput efficiency of CSMA scheduling as compared to TDMA. Finally, we tune the CSMA parameters so as to maximize the throughput to the optimal order. As a result, we show that CSMA can achieve throughput as $\Omega\left(\frac{1}{\sqrt{n}}\right)$, the same order as optimal centralized TDMA, on uniform random networks. Our CSMA scheme makes use of an efficient backbone-peripheral routing scheme and a careful design of dual carrier-sensing and dual channel scheme. We also address the implementation issues of our CSMA scheme.
\end{abstract}

Index Terms-Wireless Network Capacity, Achievable Throughput, Carrier-Sensing Multi-Access (CSMA)

\section{INTRODUCTION}

An important characteristic that distinguishes wireless networks from wired networks is the presence of spatial interference, wherein the transmission between a pair of nodes can upset other transmissions in its neighborhood. Spatial interference imposes a limit on the capacity of wireless networks.

The seminal paper [10] by Gupta and Kumar revealed that the capacity of wireless networks constrained by spatial interference is upper bounded by $\mathrm{O}\left(\frac{1}{\sqrt{n}}\right)$ for $n$ number of mutually communicating nodes on a uniform random network, no matter how optimal scheduling and routing schemes are chosen. Many similar upper bounds are derived for more sophisticated settings (e.g., with optimal source and network coding schemes [17]). In [5], Dai and Lee derive the upper bound $\mathrm{O}\left(\frac{1}{\sqrt{n}}\right)$ for multi-hop random access networks by using a simple queuing analytical argument. They also show that this upper bound is achievable only if the maximun throughput of each local node is a constant independent of $n$.

C.-K. Chau is supported by a Croucher Foundation Fellowship. M. Chen and S. C. Liew are supported by Competitive Earmarked Research Grants (Project \# 411008, 411209, and 414507) established under the University Grant Committee of the Hong Kong SAR, China. M. Chen is also supported by a Direct Grant (Project \# 2050397) of The Chinese University of Hong Kong (CUHK). Major part of the work was done when C.-K. Chau visited CUHK, under the support of the Direct Grant (Project \# 2050397) of CUHK. A preliminary version was presented at ACM MobiCom 09'.

C.-K. Chau is with University College London and University of Cambridge (e-mail: chi-kin.chau@cl.cam.ac.uk).

M. Chen and S.-C. Liew are with Department of Information Engineering at the Chinese University of Hong Kong, Shatin, Hong Kong, China (e-mail: \{minghua,soung\}@ie.cuhk.edu.hk).
Since then, a number of solutions have been proposed to achieve the upper bounds in various settings. Particularly, [7] showed that by an efficient backbone-peripheral routing scheme (analogously called "highway system") and a twostage TDMA scheme, $\Omega\left(\frac{1}{\sqrt{n}}\right)$ is achievable on a uniform random network with high probability.

So far, the studies of achievable wireless capacity in the literature consider only centralized controls and a-priori scheduling schemes with TDMA. On the practical front, carrier-sensing multi-access (CSMA) networks (e.g., Wi-Fi), which make use of distributed random-access medium-access protocols, are receiving wide adoption across enterprises and homes. It is not clear whether the results related to centrallyscheduled networks are directly applicable to CSMA networks.

To bridge the gap between practice and research, it will be interesting to find out to what extent the capacity of CSMA networks can be scaled. In particular, can the simple distributed scheduling of CSMA scales network capacity as well as central scheduling can?

The answer, according to our study, is "yes". However, the way to go about achieving CSMA scalability is non-trivial and several mechanisms must be in place before scalability can be attained. For example, the use of dual carrier-sensing power thresholds in two channels will be needed; and one must be able to assign different back-off countdown times to different nodes in a distributed manner.

To establish our results, besides building on the past work of others, we find it necessary to clarify and add rigor to the previous frameworks. It is well known that spatial interference imposes a constraint on the links that can be active simultaneously. Given an interference model, in general there can be a number of subsets of links that can be active simultaneously. Each such subset of links is called a feasible state. For a central scheduler, all feasible states are available for the design of its schedule 1 . For CSMA networks, its distributed nature does not allow us to dictate which particular feasible state will be active at what time. The problem becomes even more challenging because if not designed properly, CSMA may allow a subset of links that is not interference-safe to transmit simultaneously, leading to the so-called hidden-node problem.

We define the feasible states allowed by the CSMA protocol in a rigorous manner. We argue that the hidden-node problem in CSMA networks is caused by a mismatch between the feasible states allowed by CSMA and the feasible states of an underlying interference model. We show how to resolve this mismatch to create hidden-node free CSMA networks. Most importantly, we show that hidden-node free CSMA networks can achieve the same scaling of throughput as the central scheduler provided the above dual carrier-sensing

\footnotetext{
${ }^{1} \mathrm{~A}$ schedule is a sequence of feasible states that are active at different times.
} 
and dual channel scheme is in place. Our capacity-optimal CSMA scheme not only demonstrates the theoretical achievable throughput of CSMA networks, but also provides an implementable solution by the more practical CSMA protocols.

\section{BACKGROUND AND OVERVIEW}

The basic idea of CSMA is that before a transmitter attempts its transmission, it needs to infer the channel condition by sensing the channel. If it infers that its transmission will upset (or be upset by) any receiver's on-going transmissions (including its own receiver), then it defers its transmission. Even if the channel is sensed to be suitable for transmission, the transmitter needs to rely on a random collision avoidance mechanism, in which the transmitter initializes a random count-down period before transmission. The count-down will be frozen when channel is sensed to be not interference-safe (i.e., transmission is collision-prone), and will be resumed when the channel is sensed to be interference-safe again. A transmission will be considered successful, when the transmitter can receive an ACK packet by the corresponding receiver, upon the completion of transmission.

Compared to the centralized TDMA scheme, the CSMA protocol has two distinguishing characteristics:

i) CSMA is an ACK-based protocol, such that the receivers are required to reply an ACK packet for each successful transmission. Thus, bi-directional communications need to be explicitly considered when formulating the constraints on simultaneous transmission imposed by CSMA. The centralized TDMA schemes in prior work [7], [10], [13], [17], however, did not consider bidirectional communications and ACK packets.

ii) CSMA is a distributed random access protocol. Each transmitter chooses a random time instance to initiate its transmission, and it can only rely on its limited local knowledge to infer whether its transmission is compatible with other simultaneous transmission under various interference setting 2 . Such a distributed control requires only limited a-priori coordination among transmitters and receivers (unlike the centralized TDMA schemes).

Despite the popularity of CSMA protocols, capacity analyses applicable to large-scale CSMA wireless networks receive relatively little attention in the literature. A likely reason could be that CSMA protocols are generally regarded as synonymous to the so-called "protocol model" in many TDMA based papers. The "protocol model" is, in fact, a simplified pairwise interference model that serves to model interference among simultaneous links, which neither explicitly considers nor precisely models the aforementioned characteristics i)-ii) of CSMA 3 . There are other variants of "protocol models" in the literature [1] that also model interference among simultaneous links, rather than distributed multi-access protocols. As such, it is not clear 1) whether the capacity results based on these interference models can apply to CSMA networks; and 2)

\footnotetext{
${ }^{2}$ Note that the interference is not necessarily symmetric - a transmission could upset another simultaneous transmission but not the converse.

${ }^{3}$ Gupta and Kumar's seminal paper [10] appears to be the first to coin the phrase "protocol model", but without specifying any distributed protocol that can implement the protocol model, other than centralized schemes by TDMA.
}

whether CSMA can achieve the same throughput performance as centralized TDMA.

There is considerably large body of literature about singlehop CSMA networks [11], [14], whereas here we study the more general multi-hop CSMA networks, the results of which are quite limited in the literature [9], [15]. We also note that [3] has studied the capacity of multi-hop Aloha networks. However, Aloha protocol is different from CSMA protocol as it has no carrier-sensing operations. Also, the definition of capacity in [3] appears different from the conventional GuptaKumar's one [7], [10], [13], [17], because [3] does not require full connectivity between every pair of source and sink.

\section{A. Outline of Our Results}

To explore the capacity of CSMA networks, we first formulate the models of CSMA protocols that take into account characteristics i)-ii). These models determine the upper and lower bound on the capacity of CSMA networks, and are functions of various CSMA parameters. We then study the throughput efficiency of CSMA relative to TDMA, following the same procedure as in [14] and [12]. Finally we tune the CSMA parameters so that the capacity of a CSMA network is maximized. Our approach is divided into four parts:

1) Formulation of Carrier-sensing Decision Model (Sec. [III): Our models for CSMA protocol consist of two components that capture two major functionalities of CSMA.

- The decision model that formally formulates the constraints on simultaneous links imposed by CSMA carrier sensing operations, such as power-threshold-based carrier sensing. We explicitly distinguish the decision model of CSMA protocols from the interference model. For instance, two simultaneous links are allowed by CSMA does not necessarily mean they do not interfere with each other. This is the well-known hidden node problem.

- The random access scheme that captures how CSMA access the wireless air time and space. The key challenge is to understand the throughput efficiency of distributed channel access mechanism of CSMA, as compared to centralized TDMA scheme.

We establish the relationship between our CSMA models and the interference models from the literature in Sec. III.

2) Hidden-node-free Design of CSMA Networks (Sec. IV): There are various interference models in the literature (including the so-called "protocol model"). They are intended to capture uni-directional transmissions where ACK packets are not required. In this paper, we extend these interference models to the setting of bi-directional transmissions, under which CSMA protocols typically operate.

It is well-known that the local distributed controls of transmissions in CSMA may not be able to prevent spatial interference, as known as the hidden node problem [11]. Utilizing our proposed carrier-sensing decision models, we formally define the hidden node problem as due to a carrier-sensing decision model violating the feasibility of a bi-directional interference model. Furthermore, we derive sufficient conditions for our carrier-sensing decision models to eliminate the hidden node problem under various interference settings (Theorem 1). Our results include the prior one in [11] as a special case. 


\begin{tabular}{|c|c|c|c|c|c|c|}
\hline & \multicolumn{2}{|c|}{ Uni-directional feasible family } & \multicolumn{2}{|c|}{ Bi-directional feasible family } & \multicolumn{2}{|c|}{ Carrier-sensing feasible family } \\
\hline & $\begin{array}{c}\text { Pairwise } \\
\text { interference }\end{array}$ & $\begin{array}{c}\text { Aggregate } \\
\text { interference }\end{array}$ & $\begin{array}{c}\text { Pairwise } \\
\text { interference }\end{array}$ & $\begin{array}{c}\text { Aggregate } \\
\text { interference }\end{array}$ & $\begin{array}{c}\text { Pairwise } \\
\text { carrier-sensing }\end{array}$ & $\begin{array}{c}\text { Aggregate } \\
\text { carrier-sensing }\end{array}$ \\
\hline $\begin{array}{l}\text { Random } \\
\text { network } \\
\text { capacity }\end{array}$ & \multicolumn{2}{|c|}{$\begin{array}{c}\text { Upper bound: } O\left(\frac{1}{\sqrt{n}}\right)[10] \\
\text { Achievable as: } \Omega\left(\frac{1}{\sqrt{n}}\right) \\
\text { by TDMA [7] }\end{array}$} & \multicolumn{2}{|c|}{$\begin{array}{c}\text { Upper bound: } O\left(\frac{1}{\sqrt{n}}\right) \text { (this paper) } \\
\text { Achievable as: } \Omega\left(\frac{1}{\sqrt{n}}\right) \\
\text { by TDMA (this paper) }\end{array}$} & \multicolumn{2}{|c|}{$\begin{array}{l}\text { Upper bound: } O\left(\frac{1}{\sqrt{n}}\right) \text { (this paper) } \\
\text { Achievable as: } \Omega\left(\frac{1}{\sqrt{n}}\right) \\
\text { by dual carrier sensing (this paper) }\end{array}$} \\
\hline
\end{tabular}
TABLE I

CAPACITY OF UNIFORM RANDOM NETWORKS OVER VARIOUS FEASIBLE FAMILIES

By eliminating the hidden-node problem, we can apply elegant mathematical tools to analyze the capacity and throughput performance of multi-hop CSMA networks.

3) Stationary State Analysis of Random Access (Sec. V): To study the behavior of the random access scheme, we consider an idealized version of IEEE 802.11 DCF based on a continuous-time Markov chain model in order to capture the essential features of CSMA. This continuous Markov chain model has already been used in various analyses [12], [14].

Based on the hidden-node-free design of CSMA networks, the long-term throughput of CSMA with random access is characterized by the stationary distribution of the continuoustime Markov chain model. Following the same procedure as in [12], [14], we present the stationary distribution, and hence, the long-term throughput of hidden-node-free CSMA networks under various carrier-sensing decision models in Sec. $\mathrm{V}$ We also show that CSMA random access schemes can be tuned to perform as well as TDMA schemes.

4) Design of Dual Carrier-Sensing (Secs. VI|VII): On hidden-node-free CSMA networks, we show that the current CSMA setting with a single homogeneous carrier-sensing operation fails to achieve the optimal capacity $\Omega\left(\frac{1}{\sqrt{n}}\right)$ on a uniform random network. It can at most achieve a capacity of $\mathrm{O}\left(\frac{1}{\sqrt{n \log n}}\right)$ with high probability (shown by Theorem 2).

We then show that the design of dual carrier-sensing operations can achieve the capacity of the same order as optimal centralized TDMA. Our design is drawn from an efficient backbone-peripheral routing scheme in [7], based on which we show two different carrier-sensing power thresholds are sufficient to achieve optimal capacity of $\Omega\left(\frac{1}{\sqrt{n}}\right)$ on a uniform random network with high probability (shown by Theorem 3 ).

Not only have we provided insights in this paper for the optimal asymptotic capacity of wireless networks by our dual carrier-sensing scheme, but also addressed practical issues of implementing our scheme. First, we address the scalability issue during the dynamic switching between the dual carriersensing operations. We propose to use two frequency channels to distinguish the two carrier-sensing operations. Second, we address the issue of half-duplexity across two frequency channels, which enables low-cost implementation of our scheme.

We summarize our results and related work in Table \.

\section{Formulation AND Models}

First, note that some key notations are listed in Table III.

A central problem of multi-hop wireless communications is defined as follows. Given a set of source-sink pairs $N^{\text {sd }}$ and a set of data rate $\left\{\lambda_{k}, k \in N^{\text {sd }}\right\}$, we ask whether successful wireless communications can be established between all the sources and sinks in $N^{\text {sd }}$ to sustain the required rate $\left\{\lambda_{k}, k \in\right.$ $\left.N^{\text {sd }}\right\}$, possibly using other nodes as relays, subject to a certain interference model of simultaneous wireless transmissions.
TABLE II

KEY NOTATIONS

\begin{tabular}{|c|c|}
\hline Notation & Definition \\
\hline$N^{\mathrm{sd}}$ & set of source-sink pairs. \\
\hline$\lambda_{k}$ & data rate of source-sink pair $k \in N^{\text {sd }}$. \\
\hline$X$ & $\begin{array}{l}\text { set of relaying links induced by the paths } \\
\text { between all source-sink pairs in } N^{\text {sd }} \text {. }\end{array}$ \\
\hline$t_{i}$ & coordinates of the transmitter of link $i \in X$. \\
\hline$r_{i}$ & coordinates of the receiver of link $i \in X$. \\
\hline $\mathcal{S}$ & $\begin{array}{l}\text { feasible state, a subset of links that } \\
\text { can simultaneously transmit. }\end{array}$ \\
\hline $\mathscr{F}, \mathscr{U}, \mathscr{B}, \mathscr{C}$ & feasible family, a set of feasible states. \\
\hline $\mathrm{P}_{\mathrm{tx}}$ & fixed transmission power of all nodes. \\
\hline $\mathrm{N}_{0}$ & fixed noise power. \\
\hline$\alpha$ & power decaying factor in radio transmission. \\
\hline$\beta$ & $\begin{array}{l}\text { minimum Signal-to-Interference-Noise ratio } \\
\text { for successful receptions. }\end{array}$ \\
\hline$\Delta$ & $\begin{array}{l}\text { guard-zone coefficient, used in noise-absence } \\
\text { pairwise SIR interference model. }\end{array}$ \\
\hline$r_{x c l}$ & $\begin{array}{l}\text { interference range, used in fixed range } \\
\text { interference models. }\end{array}$ \\
\hline$r_{t x}$ & $\begin{array}{l}\text { communication range, used in fixed range } \\
\text { interference models. }\end{array}$ \\
\hline$r_{\mathrm{CS}}$ & $\begin{array}{l}\text { carrier sensing range, used in pairwise } \\
\text { CSMA decision models. }\end{array}$ \\
\hline$t_{c x}$ & $\begin{array}{l}\text { carrier sensing power threshold, used in } \\
\text { aggregate CSMA decision models. }\end{array}$ \\
\hline
\end{tabular}

Specifically, we consider the following two degrees of freedom in establishing the wireless communications:

1) Routing scheme that selects the appropriate relaying nodes to connect the sources and sinks.

2) Scheduling scheme that assigns (deterministically or randomly) the opportunities of transmissions at relaying nodes.

Furthermore, these wireless communications should be established in a distributed manner with minimal global knowledge and coordination among the nodes.

Hence, we first present several common interference models of feasible simultaneous wireless transmissions. Then we extend these interference models to the setting of bi-directional communications. Next, we formulate carrier-sensing decision models that capture distributed control of transmissions.

\section{A. Interference Models}

An interference model is defined by its interference-safe feasible family. Some common interference-safe feasibility families in the literature are defined as follows.

a.0) Pairwise fixed-range feasible family: $\mathcal{S} \in \mathscr{U}_{\mathrm{fr}}^{\mathrm{pw}}\left[X, \mathrm{r}_{\mathrm{xcl}}, \mathrm{r}_{\mathrm{tx}}\right]$, if and only if for all $i, j \in \mathcal{S}$,

$$
\left|t_{j}-r_{i}\right| \geq r_{\mathrm{xcl}} \text { and }\left|t_{i}-r_{i}\right| \leq \mathrm{r}_{\mathrm{tx}}
$$

a.1) Pairwise (noise-absent) SIR feasible family: $\mathcal{S} \in \mathscr{U}_{\mathrm{sir}}^{\mathrm{pw}}[X, \Delta]$, if and only if for all $i, j \in \mathcal{S}$,

$$
\left|t_{j}-r_{i}\right| \geq(1+\Delta)\left|t_{i}-r_{i}\right|
$$

a.2) Pairwise SINR feasible family: 
$\mathcal{S} \in \mathscr{U}_{\mathrm{sinr}}^{\mathrm{pw}}[X, \beta]$, if and only if for all $i, j \in \mathcal{S}$,

$$
\frac{\mathrm{P}_{\mathrm{tx}}\left|t_{i}-r_{i}\right|^{-\alpha}}{\mathrm{N}_{0}+\mathrm{P}_{\mathrm{tx}}\left|t_{j}-r_{i}\right|^{-\alpha}} \geq \beta
$$

a.3) Aggregate SINR feasible family:

$\mathcal{S} \in \mathscr{U}_{\operatorname{sinr}}^{\text {ag }}[X, \beta]$, if and only if for all $i \in \mathcal{S}$,

$$
\frac{\mathrm{P}_{\mathrm{tx}}\left|t_{i}-r_{i}\right|^{-\alpha}}{\mathrm{N}_{0}+\sum_{j \in \mathcal{S} \backslash\{i\}} \mathrm{P}_{\mathrm{tx}}\left|t_{j}-r_{i}\right|^{-\alpha}} \geq \beta
$$

We assume $r_{\mathrm{xcl}}>\mathrm{r}_{\mathrm{tx}}, \Delta>0, \alpha>2, \beta>0$, and uniform power $\mathrm{P}_{\mathrm{tx}}$ at all nodes. For a.2)-a.3), we assume $\mathrm{P}_{\mathrm{tx}} \mid t_{i}-$ $\left.r_{i}\right|^{-\alpha} \geq \beta \mathrm{N}_{0}$ for all $i \in X$. Otherwise, $t_{i}$ cannot successfully transmit packets to $r_{i}$ even without interference.

The notion of feasible family generalizes the notion of conflict graph implicit in a.0)-a.2), in which a feasible state is an independent set of the conflict graph arising from the model. a.0)-a.2) can serve as approximations to the more realistic interference-safe constraint a.3), when path-loss exponent $\alpha$ is large and background noise $\mathrm{N}_{0}$ is small.

In [10], pairwise SIR interference model a.1) is called "protocol model", whereas aggregate SINR interference model a.3) is called "physical model". The naming in this paper emphasizes the interference of transmissions, and avoids confusion with CSMA protocol models 4 .

\section{B. Bi-directional Interference Models}

The interference-safe constraints a.0)-a.3) are unidirectional, based on the assumption that the receiver is not required to reply an ACK packet to the transmitter upon a successful transmission. For ACK-based transmissions, interference can occur between two transmitters, between two receivers, and between a transmitter and a receiver. See Fig. 11 for an example of pairwise SIR interference model. Without the reception of ACK packets, the transmitter will consider the transmission unsuccessful and retransmit the DATA packet later on. Hence, we need to ensure that the transmissions of DATA packets and ACK packets of all simultaneous links do not interfere with each other.

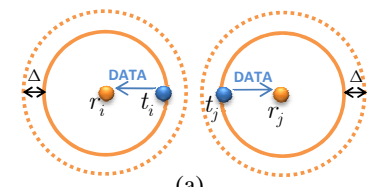

(a)

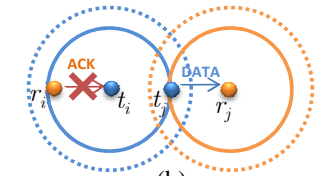

(b)
Fig. 1. In Fig. (a) the normal DATA packet transmissions from transmitters will not interfere with each other, but in Fig. (b) there is interference when transmitting ACK packet.

Let $\operatorname{dist}(i, j) \triangleq \min \left(\left|t_{j}-r_{i}\right|,\left|r_{j}-t_{i}\right|,\left|r_{j}-r_{i}\right|,\left|t_{j}-t_{i}\right|\right)$. We consider the bi-directional versions of interference-safe constraints as follows.

b.0) Bi-directional pairwise fixed-range feasible family: $\mathcal{S} \in \mathscr{B}_{\mathrm{fr}}^{\mathrm{pw}}\left[X, \mathrm{r}_{\mathrm{xcl}}, \mathrm{r}_{\mathrm{tx}}\right]$, if and only if for all $i, j \in \mathcal{S}$,

$$
\operatorname{dist}(i, j) \geq \mathrm{r}_{\mathrm{xcl}} \text { and }\left|t_{i}-r_{i}\right| \leq \mathrm{r}_{\mathrm{tx}}
$$

${ }^{4}$ We remark that [1] also presents a "generalized protocol model" with arbitrary interference footprint around the transmitters that models more general pairwise interference settings, and a "generalized physical model" that specifically applies to the Gaussian channel. b.1) Bi-directional pairwise SIR feasible family: $\mathcal{S} \in \mathscr{B}_{\text {sir }}^{\text {pw }}[X, \Delta]$, if and only if for all $i, j \in \mathcal{S}$,

$$
\operatorname{dist}(i, j) \geq(1+\Delta)\left|t_{i}-r_{i}\right|
$$

b.2) Bi-directional pairwise SINR feasible family: $\mathcal{S} \in \mathscr{B}_{\operatorname{sinr}}^{\mathrm{pw}}[X, \beta]$, if and only if for all $i, j \in \mathcal{S}$,

$$
\frac{\mathrm{P}_{\mathrm{tx}}\left|t_{i}-r_{i}\right|^{-\alpha}}{\mathrm{N}_{0}+\mathrm{P}_{\mathrm{tx}}(\operatorname{dist}(i, j))^{-\alpha}} \geq \beta
$$

b.3) Bi-directional aggregate SINR feasible family: $\mathcal{S} \in \mathscr{B}_{\text {sinr }}^{\text {ag }}[X, \beta]$, if and only if for all $i \in \mathcal{S}$,

$$
\frac{\mathrm{P}_{\mathrm{tx}}\left|t_{i}-r_{i}\right|^{-\alpha}}{\mathrm{N}_{0}+\sum_{j \in \mathcal{S} \backslash\{i\}} \mathrm{P}_{\mathrm{tx}}(\operatorname{dist}(i, j))^{-\alpha}} \geq \beta
$$

Compared with the uni-direction interference-safe constraints, the bi-directional counterparts consider the interference effect from both transmitters and receivers.

\section{Carrier-Sensing Decision Models}

The interference-safe constraints a.0)-a.3) and b.0)-b.3) capture the global spatial interference in the network. In CSMA, a transmitter has only local knowledge of its interference condition, but not the interference conditions at its targeted receiver or at the transmitting and receiving nodes of other active links. The decision of a transmitter whether to transmit is only determined by its carrier-sensing operation, rather than by the global knowledge of spatial interference.

We define carrier-sensing decision models, in which a feasible family is a set of links that may transmit simultaneously under a carrier sensing operation. But this feasible family may or may not be interference-safe under the uni-/bi-directional interference models. Two useful feasible families to capture common carrier-sensing operations are as follows.

c.1) Pairwise carrier-sensing feasible family: $\mathcal{S} \in \mathscr{C}^{\mathrm{pw}}\left[X, \mathrm{r}_{\mathrm{cs}}\right]$, if and only if for all $i, j \in \mathcal{S}$,

$$
\left|t_{j}-t_{i}\right| \geq \mathrm{r}_{\mathrm{cs}}
$$

c.2) Aggregate carrier-sensing feasible family: $\mathcal{S} \in \mathscr{C}^{\mathrm{ag}}\left[X, \mathrm{t}_{\mathrm{cs}}\right]$, if and only if for all $i \in \mathcal{S}$,

$$
\mathrm{N}_{0}+\sum_{j \in \mathcal{S}} \mathrm{P}_{\mathrm{tx}}\left|t_{j}-t_{i}\right|^{-\alpha} \leq \mathrm{t}_{\mathrm{cs}}
$$

Aggregate carrier-sensing decision model c.2) captures power-threshold based carrier sensing, where a transmitter decides its transmissions based on the channel sensing result. Pairwise carrier-sensing decision model c.1) can serve as an approximation to c.2), when path-loss exponent $\alpha$ is large and background noise $\mathrm{N}_{0}$ is small. Alternatively, c.1) can be carrier sensing based on hand-shaking messages, in which $r_{\mathrm{cs}}$ can be interpreted as the coverage area of an intended transmitter, such that within the distance $r_{c s}$ the hand-shaking messages (e.g., RTS, DATA) transmissions from this transmitter can be successfully sensed by other neighboring transmitters in its coverage, deterring them from transmissions. 


\section{HIDDEN-NODE-FREE DESIGN}

Using only local interference conditions, the local decisions of transmissions in CSMA cannot completely prevent harmful spatial interference (i.e., the hidden-node problem), or may sometimes over-react to benign spatial interference (i.e., the exposed-node problem). While they are well recognized in the literature, lacking are formal definitions that comprehensively consider various interference and carrier-sensing decision models. Here, we provide formal definitions to hiddennode and exposed-node problems based on the models in $\mathrm{Sec}$ III We then also provide sufficient conditions to eliminate the hidden-node problem.

Because CSMA is an ACK-based protocol, we consider a bi-directional interference-safe feasible family $\mathscr{B}[X]$ from one of b.0)-b.3). Given a carrier-sensing feasible family $\mathscr{C}[X]$ from one of c.1)-c.2), we define

- Hidden-node problem: if $\mathscr{B}[X] \nsupseteq \mathscr{C}[X]$

- Exposed-node problem: if $\mathscr{C}[X] \nsupseteq \mathscr{B}[X]$

Namely, hidden-node problem refers to situations where the carrier-sensing decision violates the bi-directional interference-safe constraints, whereas exposed-node problem is where the carrier-sensing decision is overly conservative in attempting to conform to the bi-directional interference-safe constraints. Our definitions naturally generalize the ones in [11], which considers only pairwise interference and carriersensing decision models. For example, we illustrate an instance of hidden-node problem for pairwise carrier-sensing decision model and pairwise SIR interference model in Fig. 2 .

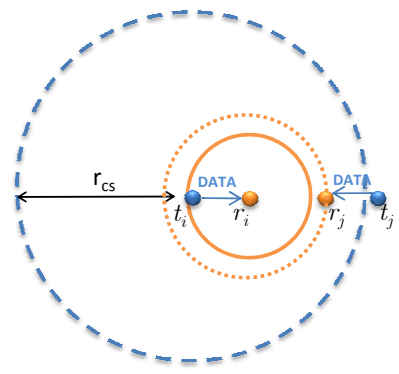

(a)

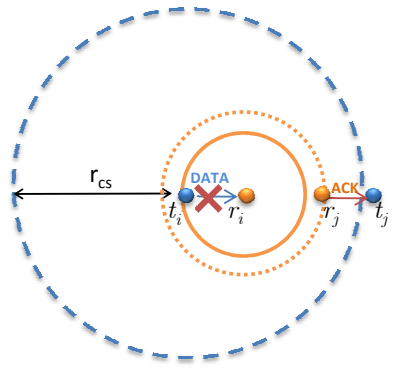

(b)
Fig. 2. In Fig. (a) the carrier-sensing decision model correctly permits the simultaneous links for DATA packet transmission, but fails in the case of ACK packet transmission in Fig. (b). Hence, $\mathscr{B}_{\text {sir }}^{\text {pw }}[X] \nsupseteq \mathscr{C}^{\text {pw }}[X]$

As studied in [11], hidden-node problem causes unfairness in CSMA networks. In this paper, we only consider CSMA networks that are designed to be hidden-node free. Besides the benefit of better fairness, more importantly, the overall performance of a hidden-node free CSMA network is tractable analytically. For example, the crucial Eqn. (23) of CSMA stationary states to be presented in Sec. $\mathrm{V}$ is valid only for a CSMA network that is hidden-node free.

One of our contributions is to establish a comprehensive set of sufficient conditions to eliminate hidden-node problem in various interference and carrier-sensing decision models. The basic idea is to establish a set of subset-relationships among all the feasible families of interference and carrier-sensing decision models in the following. Hence, under some suitable settings, a carrier-sensing feasible family can be configured as a subset to a given interference-safe feasible family, thus eliminating the hidden-node problem altogether.

Lemma 1: If $\Delta \leq \beta^{\frac{1}{\alpha}}-1$, then

$$
\mathscr{U}_{\text {sir }}^{\mathrm{pw}}[X, \Delta] \supseteq \mathscr{U}_{\operatorname{sinr}}^{\mathrm{pw}}[X, \beta] \supseteq \mathscr{U}_{\operatorname{sinr}}^{\mathrm{ag}}[X, \beta]
$$

Lemma 2: Let $r_{\mathrm{tx}}=\max _{i \in X}\left|t_{i}-r_{i}\right|$. If

$$
r_{\mathrm{xcl}} \geq\left(\frac{1}{P_{\mathrm{tx}} k(\alpha)}\left(\frac{\mathrm{P}_{\mathrm{tx}}}{\beta} \mathrm{r}_{\mathrm{tx}}^{-\alpha}-\mathrm{N}_{0}\right)\right)^{-\frac{1}{\alpha}}+\mathrm{r}_{\mathrm{tx}}
$$

where $\mathrm{k}(\alpha) \triangleq \sum_{k=1}^{\infty} 2\lceil\pi(2 k+2)\rceil k^{-\alpha}$, then

$$
\mathscr{U}_{\mathrm{sinr}}^{\mathrm{ag}}[X, \beta] \supseteq \mathscr{U}_{\mathrm{fr}}^{\mathrm{pw}}\left[X, \mathrm{r}_{\mathrm{xcl}}, \mathrm{r}_{\mathrm{tx}}\right]
$$

Note that $\mathrm{k}(\alpha)$ converges rapidly to finite constant 26 , when $\alpha>2$. See Fig. 3. (a) for a plot of the numerical values of $\mathrm{k}(\alpha)$. We remark that that [8] considers the simpler aggregate noise-absent SIR model. Because of the absence of noise, using a tighter packing lattice [8] yields a tighter constant $\mathrm{k}(\alpha)$.

Lemma 3: If $r_{x c l}^{\prime} \geq r_{x c l}+2 r_{t x}$, then

$$
\mathscr{U}_{\mathrm{fr}}^{\mathrm{pw}}\left[X, \mathrm{r}_{\mathrm{xcl}}, \mathrm{r}_{\mathrm{tx}}\right] \supseteq \mathscr{B}_{\mathrm{fr}}^{\mathrm{pw}}\left[X, \mathrm{r}_{\mathrm{xcl}}, \mathrm{r}_{\mathrm{tx}}\right] \supseteq \mathscr{U}_{\mathrm{fr}}^{\mathrm{pw}}\left[X, \mathrm{r}_{\mathrm{xcl}}^{\prime}, \mathrm{r}_{\mathrm{tx}}\right]
$$

Lemma 4: If $\Delta^{\prime} \geq \Delta+2$, then

$$
\mathscr{U}_{\text {sir }}^{\mathrm{pw}}[X, \Delta] \supseteq \mathscr{B}_{\text {sir }}^{\mathrm{pw}}[X, \Delta] \supseteq \mathscr{U}_{\mathrm{sir}}^{\mathrm{pw}}\left[X, \Delta^{\prime}\right]
$$

Lemma 5: If $\beta^{\prime} \geq\left(2+\beta^{\frac{1}{\alpha}}\right)^{\alpha}$, then

$$
\mathscr{U}_{\text {sinr }}^{\mathrm{pw}}[X, \beta] \supseteq \mathscr{B}_{\operatorname{sinr}}^{\mathrm{pw}}[X, \beta] \supseteq \mathscr{U}_{\text {sinr }}^{\mathrm{pw}}\left[X, \beta^{\prime}\right]
$$

Lemma 6: If $\beta^{\prime} \geq\left(2+\beta^{\frac{1}{\alpha}}\right)^{\alpha}$, then

$$
\mathscr{U}_{\text {sinr }}^{\mathrm{ag}}[X, \beta] \supseteq \mathscr{B}_{\operatorname{sinr}}^{\mathrm{ag}}[X, \beta] \supseteq \mathscr{U}_{\text {sinr }}^{\mathrm{ag}}\left[X, \beta^{\prime}\right]
$$

Lemma 7: If $\mathrm{r}_{\mathrm{tx}}=\max _{i \in X}\left|t_{i}-r_{i}\right|$ and $\mathrm{r}_{\mathrm{cs}} \geq \mathrm{r}_{\mathrm{xcl}}+2 \mathrm{r}_{\mathrm{tx}}$,

$$
\mathscr{C}^{\mathrm{pw}}\left[X, \mathrm{r}_{\mathrm{xcl}}\right] \supseteq \mathscr{B}_{\mathrm{fr}}^{\mathrm{pw}}\left[X, \mathrm{r}_{\mathrm{xcl}}, \mathrm{r}_{\mathrm{tx}}\right] \supseteq \mathscr{C}^{\mathrm{pw}}\left[X, \mathrm{r}_{\mathrm{cs}}\right]
$$

Lemma 8: If $\mathrm{t}_{\mathrm{cs}} \leq \mathrm{N}_{0}+\mathrm{P}_{\mathrm{t} \times} \mathrm{r}_{\mathrm{cs}}^{-\alpha}$, then

$$
\mathscr{C}^{\mathrm{pw}}\left[X, \mathrm{r}_{\mathrm{cs}}\right] \supseteq \mathscr{C}^{\mathrm{ag}}\left[X, \mathrm{t}_{\mathrm{cs}}\right]
$$

Lemma 9: If $\mathrm{r}_{\mathrm{cs}} \geq\left(\frac{1}{\mathrm{P}_{\mathrm{tx}} \mathrm{k}(\alpha)}\left(\mathrm{t}_{\mathrm{cs}}-\mathrm{N}_{0}\right)\right)^{-\frac{1}{\alpha}}$, then

$$
\mathscr{C}^{\mathrm{ag}}\left[X, \mathrm{t}_{\mathrm{cs}}\right] \supseteq \mathscr{C}^{\mathrm{pw}}\left[X, \mathrm{r}_{\mathrm{cs}}\right]
$$

Note that Lemma 4 can be proven by applying Lemma 5 and letting $\mathrm{N}_{0}=0, \Delta=\beta^{\frac{1}{\alpha}}-1$ and $\Delta^{\prime}=\beta^{\prime \frac{1}{\alpha}}-1$. Hence, $(2+$ $\left.\beta^{\frac{1}{\alpha}}\right)^{\alpha}$ is a universal constant for both pairwise and aggregate interference models with/without noise. See Fig. 3. (b) for a plot of the numerical values of $\left(2+\beta^{\frac{1}{\alpha}}\right)^{\alpha}$.

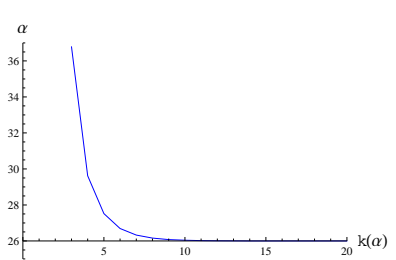

(a)

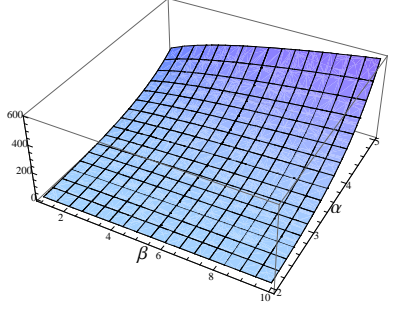

(b)
Fig. 3. Fig. (a): Numerical values of $\mathrm{k}(\alpha)$, which converges rapidly to finite constant 26 when $\alpha>2$. Fig. (b): Numerical values of $\left(2+\beta^{\frac{1}{\alpha}}\right)^{\alpha}$. 


\section{A. Hidden-node-free Sufficient Conditions}

Lemmas 18 establish a tree diagram Fig. 4 of subsetrelationships for the interference and carrier-sensing decision models, under the respective sufficient conditions.

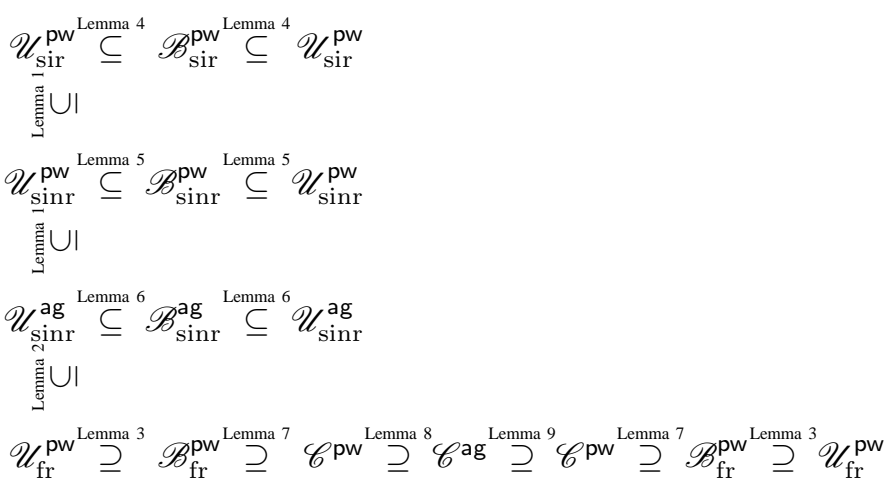

Fig. 4. The tree diagram represents the subset-relationships for the interference and carrier-sensing decision models.

The tree diagram Fig. 4 provides us a way to design hidden-node-free CSMA networks. Given any bi-directional interference-safe feasible family $\mathscr{B}[X]$ from b.0)-b.3), and any carrier-sensing feasible family $\mathscr{C}[X]$ from c.1)-c.2), we start at $\mathscr{B}[X]$ in the tree diagram, and follow the respective chains of lemmas to set the respective sufficient conditions until reaching $\mathscr{C}[X]$. Then, we can obtain a hidden-node-free design. Hence, it proves the following theorem.

Theorem 1: Suppose $r_{\mathrm{tx}}=\max _{i \in X}\left|t_{i}-r_{i}\right|$. For any bidirectional interference-safe feasible family $\mathscr{B}[X]$ from b.0)b.3) and any carrier-sensing feasible family $\mathscr{C}[X]$ from c.1)c.2), there exists a suitable setting of $r_{c s}$ or $t_{c s}$ such that

$$
\text { (Hidden-node-free Design) : } \mathscr{B}[X] \supseteq \mathscr{C}[X]
$$

We summarize the sufficient conditions for hidden-nodefree CSMA network design in Table III]

Although the virtual carrier sensing (RTS/CTS) in IEEE 802.11 is designed to solve the hidden node problem, using RTS/CTS in multi-hop networks does not eliminate the hidden-node problem [16], unless the carrier sensing range is large enough and a number of other conditions are met [11]. The conditions for hidden-node free operation under the RTS/CTS mode are much more complicated than under the basic mode, even under the pairwise interference model (see [11] for details). To keep our focus in this paper, we will not consider the RTS/CTS mode. The extension to incorporate RTS/CTS is certainly an interesting subject for future studies, particularly for the hidden-node free operation under the aggregate interference model. Moreover, Theorem 11 covers hidden-node free operation of the basic mode under the aggregate interference model, which was not treated in [11].

\section{STATIONARY THROUGHPUT ANALYSIS}

While Sec. III IV address the distributed and ACK-based nature of CSMA, this section addresses the characteristics of random access in CSMA, and study its achievable capacity as compared to TDMA schemes.

\section{A. Deterministic Scheduling}

Consider a given routing scheme and a carrier-sensing feasible family $\mathscr{C}[X]$ (that is set to be hidden-node-free by Theorem [1). If we assume slotted time, a deterministic scheduling scheme is defined as a sequence $\left(\mathcal{S}_{\mathrm{t}}\right)_{\mathrm{t}=1}^{\mathrm{m}}$ where each $\mathcal{S}_{\mathrm{t}} \in \mathscr{C}[X]$, such that the transmitters in each $\mathcal{S}_{\mathrm{t}}$ are allowed to transmit only at every timeslot $(\mathrm{t} \bmod \mathrm{m})$. A TDMA scheme is simply a deterministic scheduling scheme. Such a TDMA scheme is only a hypothetical scheme that can serve as a "reference" scheme for the study of the random access based CSMA network.

Suppose the bandwidth is normalized to a unit constant. Then for each link $i \in X$, the throughput rate under scheduling scheme $\left(\mathcal{S}_{\mathrm{t}}\right)_{\mathrm{t}=1}^{\mathrm{m}}$ is:

$$
\mathfrak{c}_{i}^{\operatorname{det}}\left[\left(\mathcal{S}_{\mathrm{t}}\right)_{\mathrm{t}=1}^{\mathrm{m}}\right] \triangleq \frac{1}{\mathrm{~m}} \sum_{\mathrm{t}=1}^{\mathrm{m}} \mathbb{1}\left(i \in \mathcal{S}_{\mathrm{t}}\right)
$$

Recall $\lambda_{k}$ is the date rate of source-sink pair $k \in N^{\text {sd }}$. With the routing scheme, one can determine the feasible region for $\left(\lambda_{k}\right)_{k \in N^{\text {sd }}}$ by solving a multi-commodity flow problem.

\section{B. Multi-Backoff-Rate Random Access}

More generally, we consider a random access scheme (e.g., IEEE 802.11 DCF), such that $\left(\mathcal{S}_{\mathrm{t}}\right)_{\mathrm{t}=1}^{\infty}$ follows a random sequence. We consider an idealized version CSMA random access scheme as a continuous-time Markov process as in [6], [12], [14], which is sufficient to provide insights for the practical CSMA random access scheme. We assume that the count-down time and transmission time follow exponential distribution 5 . The average count-down time can be distinct for different links. Thus, we call this multi-backoff-rate random access. We formalize the random access scheme by a Markov chain with its states being $\mathscr{C}[X]$. There is a possible transition between states $\mathcal{S}, \mathcal{S}^{\prime} \in \mathscr{C}[X]$, if $\mathcal{S}=\{i\} \cup \mathcal{S}^{\prime}$ for some $i \in X$. Suppose $\mathcal{S} \in \mathscr{C}[X]$ and $(\{i\} \cup \mathcal{S} \in \mathscr{C}[X]$ or $\{i\} \cup \mathcal{S} \in \mathscr{C}[X]$ ).

- Transition " $\mathcal{S} \rightarrow\{i\} \cup \mathcal{S}$ " represents that the transmitter of link $i$ will start to transmit, after some random countdown time.

- Transition " $\{i\} \cup \mathcal{S} \rightarrow \mathcal{S}$ " represents that the transmitter of link $i$ will finish transmission, after some random transmission time.

Suppose the current state of simultaneous transmissions is $\mathcal{S}$, and transmitter $i$ is counting down to transmission. $i$ will freeze count-down if it detects that the channel is busy (i.e., $\mathcal{S} \rightarrow\{j\} \cup \mathcal{S}$ for some $j \neq i$, and $\{i, j\} \cup \mathcal{S} \notin \mathscr{C}[X]$ ). $i$ will resume count-down until the state of simultaneous transmissions becomes $\mathcal{S}^{\prime}$ such that $\{i\} \cup \mathcal{S}^{\prime} \in \mathscr{C}[X]$.

Let the rate of transition $\mathcal{S} \rightarrow\{i\} \cup \mathcal{S}$ be $\nu_{i}$, and normalize the rate of transition $\{i\} \cup \mathcal{S} \rightarrow \mathcal{S}$ as 1 . Let $\nu \triangleq\left(\nu_{i}\right)_{i \in X}$. Then $\langle\mathscr{C}[X], \nu\rangle$ denotes the continuous-time Markov process of idealized multi-backoff-rate CSMA random access.

\footnotetext{
${ }^{5} \mathrm{~A}$ main result upon which the results of this paper is built is the stationary probability distribution in Eqn. 23. It turns out that Eqn. 23] is insensitive to the distributions of the count-down and transmission times. That is, Eqn. 23 is still the stationary probability distribution under general transmission-time distribution and general count-down time distribution with memory.
} 


\begin{tabular}{|c|c|c|c|c|}
\hline & \multicolumn{4}{|c|}{ Bi-directional feasible family } \\
\hline & $\begin{array}{l}\text { pairwise fixed range } \\
\mathscr{B}_{\mathrm{fr}}^{\mathrm{pw}}\left[X, \mathrm{r}_{\mathrm{xcl}}, \mathrm{r}_{\mathrm{tx}}\right] \\
\end{array}$ & $\begin{array}{l}\text { pairwise SIR } \\
\mathscr{B}_{\text {sir }}^{\mathrm{pw}}[X, \Delta] \\
\end{array}$ & $\begin{array}{l}\text { pairwise SINR } \\
\mathscr{B}_{\mathrm{sinr}}^{\mathrm{pw}}[X, \beta] \\
\end{array}$ & $\begin{array}{l}\text { aggregate SINR } \\
\mathscr{B}_{\operatorname{sinr}}^{\text {ag }}[X, \beta] \\
\end{array}$ \\
\hline $\begin{array}{c}\text { Pairwise } \\
\text { carrier-sensing } \\
\text { feasible family } \\
\mathscr{C}^{\text {pw }}\left[X, \mathrm{r}_{\mathrm{cs}}\right]\end{array}$ & $\begin{array}{c}\mathrm{r}_{\mathrm{tx}}=\max _{i \in X}\left|t_{i}-r_{i}\right| \\
\mathrm{r}_{\mathrm{cs}} \geq \mathrm{r}_{\mathrm{xcl}}+2 \mathrm{r}_{\mathrm{tx}}\end{array}$ & $\begin{array}{c}r_{\mathrm{tx}}=\max _{i \in X}\left|t_{i}-r_{i}\right| \\
\mathrm{r}_{\mathrm{cs}} \geq(3+\Delta) \mathrm{r}_{\mathrm{tx}}[11]\end{array}$ & $\begin{array}{c}\mathrm{r}_{\mathrm{tx}}=\max _{i \in X}\left|t_{i}-r_{i}\right| \\
\mathrm{r}_{\mathrm{cs}} \geq\left(\frac{1}{\mathrm{P}_{\mathrm{tx}}}\left(\frac{\mathrm{P}_{\mathrm{tx}}}{\left(2+\beta^{\frac{1}{\alpha}}\right)^{\alpha}} r_{\mathrm{tx}}^{-\alpha}\right.\right. \\
\left.\left.-\mathrm{N}_{0}\right)\right)^{-\frac{1}{\alpha}}+2 \mathrm{r}_{\mathrm{tx}} \quad \text { (See Lemma 19] }\end{array}$ & $\begin{array}{c}\mathrm{r}_{\mathrm{tx}}=\max _{i \in X}\left|t_{i}-r_{i}\right| \\
\mathrm{r}_{\mathrm{cs}} \geq\left(\frac{1}{\mathrm{P}_{\mathrm{tx} \mathrm{k}(\alpha)}}\left(\frac{\mathrm{P}_{\mathrm{tx}}}{\left(2+\beta^{\frac{1}{\alpha}}\right)^{\alpha}} r_{\mathrm{tx}}^{-\alpha}\right.\right. \\
\left.\left.-\mathrm{N}_{0}\right)\right)^{-\frac{1}{\alpha}}+3 \mathrm{r}_{\mathrm{tx}}\end{array}$ \\
\hline $\begin{array}{c}\text { Aggregate } \\
\text { carrier-sensing } \\
\text { feasible family } \\
\mathscr{C}^{\text {ag }}\left[X, \mathrm{t}_{\mathrm{cs}}\right]\end{array}$ & \multicolumn{4}{|c|}{$\begin{array}{l}\text { Let } r_{\mathrm{cs}} \text { be a carrier-sensing range satisfying the above corresponding conditions, } \\
\qquad t_{\mathrm{cs}} \leq \mathrm{N}_{0}+\mathrm{P}_{\mathrm{t} \times} \mathrm{r}_{\mathrm{cS}}^{-\alpha}\end{array}$} \\
\hline
\end{tabular}

SUFFICIENT CONDITIONS FOR HIDDEN-NODE-FREE CSMA NETWORK DESIGN. RESULTS ARE DERIVED IN THIS PAPER UNLESS CITED OTHERWISE.

Lemma 10: $\langle\mathscr{C}[X], \nu\rangle$ is a reversible Markov process, with stationary distribution for each $\mathcal{S} \in \mathscr{C}[X]$ as:

$$
\mathbb{P}_{\nu}(\mathcal{S})=\frac{\exp \left(\sum_{i \in \mathcal{S}} \log \nu_{i}\right)}{\sum_{\mathcal{S}^{\prime} \in \mathscr{C}[X]} \exp \left(\sum_{j \in \mathcal{S}^{\prime}} \log \nu_{j}\right)}
$$

The long-term throughput is characterized by the stationary distribution of $\langle\mathscr{C}[X], \nu\rangle$. Therefore, for each link $i \in X$, the throughput rate under idealized multi-backoff-rate CSMA random access is:

$$
\mathfrak{c}_{i}^{\text {rand }}[\langle\mathscr{C}[X], \nu\rangle] \triangleq \sum_{\mathcal{S} \in \mathscr{C}[X]: i \in \mathcal{S}} \mathbb{P}_{\nu}(\mathcal{S})
$$

We can relate the throughput of a deterministic scheduling scheme with the long-term throughput of idealized multibackoff-rate CSMA random access by the following result.

Lemma 11: Given a deterministic scheduling scheme $\left(\mathcal{S}_{\mathrm{t}}\right)_{\mathrm{t}=1}^{\mathrm{m}}$, let the fraction of time spent in $\mathcal{S} \in \mathscr{C}[X]$ be $\mathbb{P}^{\operatorname{det}}(\mathcal{S})=\frac{1}{\mathrm{~m}} \sum_{\mathrm{t}=1}^{\mathrm{m}} \mathbb{1}\left(\mathcal{S}_{\mathrm{t}}=\mathcal{S}\right)$. If $\mathbb{P}^{\operatorname{det}}(\mathcal{S})>0$ for all $\mathcal{S} \in \mathscr{C}[X]$, then there exists count-down rates $\nu$, such that for each link $i \in X$, it satisfies:

$$
\mathfrak{c}_{i}^{\operatorname{det}}\left[\left(\mathcal{S}_{\mathrm{t}}\right)_{\mathrm{t}=1}^{\mathrm{m}}\right] \leq \mathfrak{c}_{i}^{\text {rand }}[\langle\mathscr{C}[X], \nu\rangle]
$$

Lemma 11 is proven in [12] (Proposition 2). In the Appendix, we give a simplified alternate proof.

The implication of Lemma 11 is that idealized multibackoff-rate CSMA random access can be adapted to perform at least as well as a class of TDMA schemes under the same set of feasible states. Lemma 11 will be useful to show the existence of the achievable capacity of multi-backoff-rate CSMA networks, if we know the achievable capacity of the corresponding TDMA scheme on the same $\mathscr{C}[X]$.

\section{CAPACITY OF RANDOM NETWORK}

In this section, we apply the results from Sec. III V to the capacity analysis on a uniform random network. The ramification of selecting a uniform random network is to provide the simplest average-case analysis, without involving other complicated random network topology. We consider a Poisson point proces 6 of unit density on a square plane $[0, \sqrt{n}] \times[0, \sqrt{n}]$. Every node on the plane is a source or a sink that is selected uniform-randomly among all the nodes on the plane. We next define some notations:

\footnotetext{
${ }^{6}$ One can consider an alternative point process where $n$ nodes are placed on the plane by uniform distribution. But this point process converges to Poisson point process asymptotically.
}

- $\mathcal{N}_{n}^{\text {sd }}$ denotes the random set of source-sink pairs induced by the Poisson point process.

- $\mathcal{R}$ denotes a routing scheme that assigns each $k \in \mathcal{N}_{n}^{\text {sd }}$ a path, such that each hop is within the maximum transmitter-receiver distance $\left(\mathrm{P}_{\mathrm{tx}} /\left(\beta \mathrm{N}_{0}\right)\right)^{\frac{1}{\alpha}}$.

- $\mathcal{X}_{n}^{\mathcal{R}}$ denotes the random set of links induced by routing scheme $\mathcal{R}$ over $\mathcal{N}_{n}^{\text {sd }}$.

- $\mathscr{F}\left[\mathcal{X}_{n}^{\mathcal{R}}\right]$ denotes a feasible family from a.0)-c.2) over the random set of links, $\mathcal{X}_{n}^{\mathcal{R}}$.

- $\mathscr{S}\left(\mathscr{F}\left[\mathcal{X}_{n}^{\mathcal{R}}\right]\right)$ denotes the set of all possible deterministic scheduling schemes $\left\{\left(\mathcal{S}_{\mathrm{t}} \in \mathscr{F}\left[\mathcal{X}_{n}^{\mathcal{R}}\right]\right)_{\mathrm{t}=1}^{\mathrm{m}}\right\}$.

- $\lambda\left(\mathscr{F}\left[\mathcal{X}_{n}^{\mathcal{R}}\right]\right)$ denotes the minimum data rate among all the source-sink pairs in $\mathcal{N}_{n}^{\text {sd }}$, achieved by the most optimal deterministic scheduling scheme:

$$
\lambda\left(\mathscr{F}\left[\mathcal{X}_{n}^{\mathcal{R}}\right]\right) \triangleq \max _{\left(\mathcal{S}_{\mathrm{t}}\right)_{\mathrm{t}=1}^{\mathrm{m}} \in \mathscr{\mathscr { S }}\left(\mathscr{F}\left[\mathcal{X}_{n}^{\mathcal{R}}\right]\right)}\left(\min _{k \in \mathcal{N}_{n}^{\mathrm{sd}}} \lambda_{k}\right)
$$

We now define the capacity over random networks. Since $\lambda\left(\mathscr{F}\left[\mathcal{X}_{n}^{\mathcal{R}}\right]\right)$ is a random variable, we say that the capacity over $\mathcal{N}_{n}^{\text {sd }}$ has an order as $\Theta(f(n))$ with high probability (w.h.p.), if there exists finite constants $c^{\prime}>c>0$ such that

$$
\begin{aligned}
\lim _{n \rightarrow \infty} \mathbb{P}\left\{\lambda\left(\mathscr{F}\left[\mathcal{X}_{n}^{\mathcal{R}}\right]\right)\right. & =c \cdot f(n) \text { is feasible }\}=1 \\
\liminf _{n \rightarrow \infty} & \mathbb{P}\left\{\lambda\left(\mathscr{F}\left[\mathcal{X}_{n}^{\mathcal{R}}\right]\right)=c^{\prime} \cdot f(n) \text { is feasible }\right\}<1
\end{aligned}
$$

This is the conventional definition of random wireless network capacity [7], [10], [13], [17].

\section{A. Upper Bound for Single Carrier Sensing}

We first show that carrier-sensing based on c.1)-c.2) cannot achieve the optimal capacity $\Omega\left(\frac{1}{\sqrt{n}}\right)$.

Theorem 2: Consider a carrier-sensing feasible family $\mathscr{C}\left[\mathcal{X}_{n}^{\mathcal{R}}\right]$ from c.1)-c.2), for any routing scheme $\mathcal{R}$ that connects all the source-sink pairs in $\mathcal{N}_{n}^{\text {sd }}$,

$$
\lambda\left(\mathscr{C}\left[\mathcal{X}_{n}^{\mathcal{R}}\right]\right)=\mathrm{O}\left(\frac{1}{\sqrt{n \log n}}\right) \quad \text { (w.h.p.) }
$$

Proof: By Lemmas 3]7,[8, there exists a suitable $r_{x c l}$, such that $\mathscr{C}\left[\mathcal{X}_{n}^{\mathcal{R}}\right]$ can be configured as a subset of $\mathscr{U}_{\mathrm{fr}}^{\mathrm{pw}}\left[\mathcal{X}_{n}^{\mathcal{R}}, \mathrm{r}_{\mathrm{xcl}}, r_{\mathrm{tx}}\right]$. It has been shown in [10] that

$$
\lambda\left(\mathscr{U}_{\mathrm{fr}}^{\mathrm{pw}}\left[\mathcal{X}_{n}^{\mathcal{R}}, \mathrm{r}_{\mathrm{xcl}}, \mathrm{r}_{\mathrm{tx}}\right]\right)=\mathrm{O}\left(\frac{1}{\sqrt{n \log n}}\right) \quad \text { (w.h.p.) }
$$

for any routing scheme $\mathcal{R}$ that connects all the source-sink pairs in $\mathcal{N}_{n}^{\text {sd }}$. Hence, it completes the proof.

Nonetheless, [7], [17] shows that for any interference-safe feasible family from a.1)-a.3), there exists a TDMA scheme to achieve throughput as $\Omega\left(\frac{1}{\sqrt{n}}\right)$ (w.h.p.). We are thus motivated to adopt such a TDMA-based approach to CSMA networks. 


\section{B. Backbone-Peripheral Routing}

We briefly revisit the efficient routing scheme in [7] (we call backbone-peripheral routing). Partition the nodes into two classes: backbone nodes and peripheral nodes. The backbone nodes themselves are connected using only short-range links, whereas every peripheral node can reach a backbone node in one-hop transmission. The basic idea is to use shortrange backbone-backbone links whenever possible. Since short-range links generate minimal spatial interference, this increases the number of simultaneous active links, and hence the throughput.

To implement backbone-peripheral routing, we first partition the square plane $[0, \sqrt{n}] \times[0, \sqrt{n}]$ into square cells with sidelength $s_{n}$. Considering the cells as vertices, a path can be formed by connecting adjacent non-empty cells.

Lemma 12: (See [7]) There exist constants $c_{1}, c_{2}, c_{3}$ independent of $n$, such that when we set $\mathrm{s}_{n}=c_{1}$, then in every horizontal slab of $\left(\sqrt{n} / c_{1} \times c_{2} \log n / c_{1}\right)$ cells, there exist at least $c_{3} \log n$ disjoint paths between the vertical opposite sides of the plane (w.h.p.).

We build a backbone (called "highway system" in [7]) for routing on a uniform random network as follows. Select a representative node in each non-empty cell. By Lemma 12 there is a connected sub-network that spans the plane (w.h.p.), formed by connecting the representative nodes in the adjacent cells. These connected representative nodes are the backbone nodes, while the rest are the peripheral nodes. Note that the distance between two adjacent backbone nodes is at most $\sqrt{5} c_{1}$, while the distance between a peripheral node to a nearby backbone node is at most $c_{2} \log n$ (w.h.p.).

Backbone-peripheral routing scheme operates as follows. The source first uses a one-hop transmission to a backbone node, if it is a peripheral node. We control the packet load from the peripheral nodes such that each backbone node is accessed by at most by some constant number of peripheral nodes. Next, the receiving backbone node relays the packet following multihop Manhattan-routing along the adjacent backbone nodes to the respective backbone node that can transmit the packets to the sink in a single last hop. See Fig. 5 for an illustration of backbone-peripheral routing.

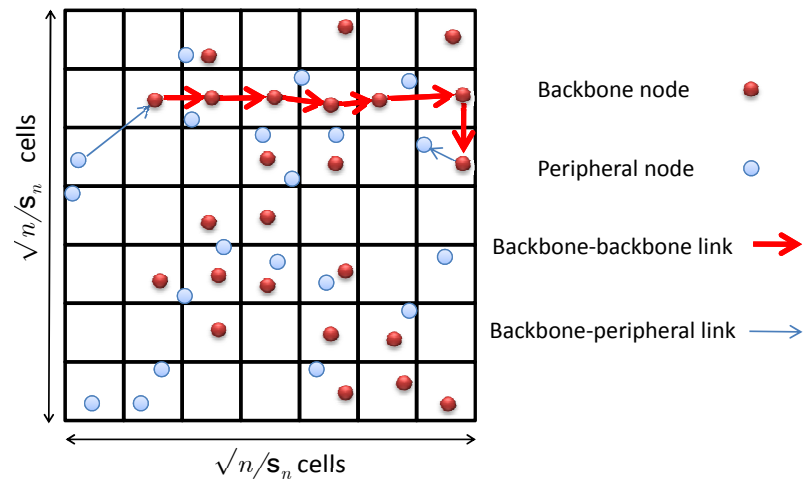

Fig. 5. Backbone nodes are a subset of connected nodes by short-range links, whereas peripheral nodes relay all the packets to backbone nodes.

We define a scheduling scheme under backbone-peripheral routing consisting of two stages: $\left(\mathcal{S}_{\mathrm{t}}^{\mathrm{P}}\right)_{\mathrm{t}=1}^{c_{4} \log ^{2} n}$ and $\left(\mathcal{S}_{\mathrm{t}}^{\mathrm{B}}\right)_{\mathrm{t}=1}^{c_{5}}$, for some constants $c_{4}, c_{5}$.
1) (Backbone-peripheral Transmissions): If $i \in \mathcal{S}_{t}^{\mathrm{P}}$, then either $t_{i}$ or $r_{i}$ is a peripheral node. Using a spatial assignment scheme, we divide the plane into larger cells, each of which having an area of $\Theta\left(\log ^{2} n\right)$ (because the backbone-peripheral distance is $\mathrm{O}(\log n))$. It is shown in [7] that we can always pick a non-interfering link in each cell to transmit in every timeslot $\left(\mathrm{t} \bmod c_{4} \log ^{2} n\right)$ in the first stage, for some constant $c_{4}$. The throughput rate for each backbone-peripheral link can be shown to be $\Theta\left(\frac{1}{\log ^{2} n}\right) \gg \Theta\left(\frac{1}{\sqrt{n}}\right)$.

2) (Backbone-backbone Transmissions): If $i \in \mathcal{S}_{\mathrm{t}}^{\mathrm{B}}$, then both $t_{i}$ and $r_{i}$ are backbone nodes. Since the backbonebackbone distance is $\mathrm{O}(1)$, we use a similar spatial assignment scheme but considering a cell with an area $c_{5}$, for some constant $c_{5}$. Since each backbone node is accessed by at most by some constant number of peripheral nodes, there are at most $\mathrm{O}(\sqrt{n})$ peripheral nodes that relays packets to each backbone node. Thus, the throughput rate at each backbone-backbone link divided by the number of peripheral nodes that relay packets to it is $\Theta\left(\frac{1}{\sqrt{n}}\right)$.

Overall, backbone-backbone links are the bottleneck, not backbone-peripheral links. Hence, $\lambda_{k}=\Omega\left(\frac{1}{\sqrt{n}}\right)$ is achievable w.h.p. on a uniform random network based on backboneperipheral routing and the above two-stage scheduling scheme.

\section{DuAl CARRIER-SENSING}

To adopt the TDMA scheme of backbone-peripheral routing in Sec. VI-B for CSMA networks, in Sec VII-A we employ dual carrier-sensing where multiple carrier-sensing ranges (or power thresholds) are allowed. Namely, smaller carrier-sensing ranges (or larger power thresholds) can be used among the short-range links. This effectively enables more simultaneous links and improves the throughput.

However, simple designs of multi-carrier sensing may not be scalable and even implementable. In Sec VII-B, we discuss some issues of implementing multi-carrier sensing. We present a careful design of dual carrier-sensing using two channels that only incurs small overhead at low implementation cost.

\section{A. Simple Designs of Dual Carrier-Sensing}

To illustrate simple designs of dual carrier-sensing, we consider pairwise carrier-sensing decision model in Fig. 6 , The basic idea is that we allow a shorter carrier-sensing range to be used among backbone-backbone links. A longer carriersensing range will be used when there is a backbone-peripheral link in the neighborhood.

Formally, we partition $X$ into two disjoint classes: $X^{\mathrm{B}}$ for backbone-backbone links, and $X^{\mathrm{P}}$ for backbone-peripheral links. Assume $r_{c s}^{B}<r_{c s}^{P}$ and $t_{c s}^{B}>t_{c s}^{P}$. Two simple dual carriersensing designs by extending c.1)-c.2) are:

d.1) Pairwise dual carrier-sensing feasible family: $\mathcal{S} \in$ $\mathscr{C}_{\mathrm{mcs}}^{\mathrm{pw}}\left[\left(X^{\mathrm{B}}, \mathrm{r}_{\mathrm{cs}}^{\mathrm{B}}\right),\left(X^{\mathrm{P}}, \mathrm{r}_{\mathrm{cs}}^{\mathrm{P}}\right)\right]$, if and only if for all $i, j \in \mathcal{S}$,

$$
\left|t_{j}-t_{i}\right| \geq \max \left\{\mathrm{r}_{\mathrm{cs}}^{\mathrm{c}}, \mathrm{r}_{\mathrm{cs}}^{\mathrm{c}^{\prime}}\right\},
$$

where $i \in X^{\mathrm{c}}, j \in X^{\mathrm{c}^{\prime}}$ and $\mathrm{c}, \mathrm{c}^{\prime} \in\{\mathrm{B}, \mathrm{P}\}$. 


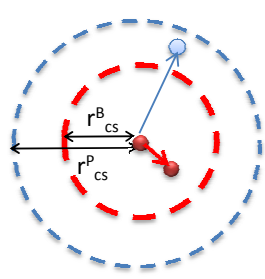

(a)
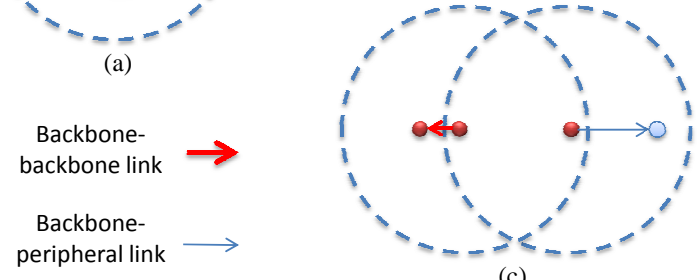

(c)

Fig. 6. There are two carrier sensing ranges as in Fig. (a). In Fig. (b) shortrange backbone-backbone links will use a shorter carrier-sensing range among themselves, while in Fig. (c) longer carrier-sensing range will used when there is any backbone-peripheral link.

d.2) Aggregate dual carrier-sensing feasible family: $\mathcal{S} \in$ $\mathscr{C}_{\mathrm{mcs}}^{\mathrm{ag}}\left[\left(X^{\mathrm{B}}, \mathrm{r}_{\mathrm{cs}}^{\mathrm{B}}\right),\left(X^{\mathrm{P}}, \mathrm{r}_{\mathrm{cs}}^{\mathrm{P}}\right)\right]$, if and only if for all $i \in \mathcal{S}$,

$$
\mathrm{N}_{0}+\sum_{j \in \mathcal{S}} \mathrm{P}_{\mathrm{tx}}\left|t_{j}-t_{i}\right|^{-\alpha} \leq \min _{\mathrm{c} \in\{\mathrm{B}, \mathrm{P}\}: X^{\mathrm{c}} \cap \mathcal{S} \neq \varnothing}\left\{\mathrm{t}_{\mathrm{cs}}^{\mathrm{c}}\right\}
$$

That is, there will be a dynamic switching process of carriersensing ranges (or power thresholds), dependent on the presence of classes of active links.

Theorem 3: Consider dual carrier-sensing decision model d.1) on a uniform random network based on backboneperipheral routing. Let $\mathcal{X}_{n}^{\mathrm{B}}$ and $\mathcal{X}_{n}^{\mathrm{P}}$ be the random set of induced backbone-backbone links and backbone-peripheral links, respectively. Using multi-backoff-rate random access scheme, there exists a suitable setting of $\left(r_{C S}^{B}, r_{C S}^{P}\right)$, such that

$$
\left.\lambda\left(\mathscr{C}_{\mathrm{mcs}}^{\mathrm{pw}}\left[\left(X^{\mathrm{B}}, \mathrm{r}_{\mathrm{cs}}^{\mathrm{B}}\right),\left(X^{\mathrm{P}}, \mathrm{r}_{\mathrm{cs}}^{\mathrm{P}}\right)\right]\right)=\Omega\left(\frac{1}{\sqrt{n}}\right) \quad \text { (w.h.p. }\right)
$$

Proof: Recall that $\left(\mathcal{S}_{\mathrm{t}}^{\mathrm{P}}\right)_{\mathrm{t}=1}^{c_{4} \log ^{2} n}$ and $\left(\mathcal{S}_{\mathrm{t}}^{\mathrm{B}}\right)_{\mathrm{t}=1}^{c_{5}}$ are the two stage TDMA schemes in backbone-peripheral routing. Note that each $\mathcal{S}_{\mathrm{t}}^{\mathrm{P}}$ and $\mathcal{S}_{\mathrm{t}}^{\mathrm{B}}$ is a feasible state in a uni-directional pairwise SIR interference model.

We set $\mathrm{r}_{\mathrm{cs}}^{\mathrm{B}}=c_{6}$ and $\mathrm{r}_{\mathrm{cs}}^{\mathrm{P}}=c_{7} \log n$ for some constants $c_{6}, c_{7}$. Then according to Theorem 1 we can obtain some constants $c_{6}, c_{7}$, such that

$$
\begin{gathered}
\mathcal{S}_{\mathrm{t}}^{\mathrm{P}} \subseteq \mathscr{C}_{\mathrm{mcs}}^{\mathrm{pw}}\left[\left(X^{\mathrm{B}}, \mathrm{r}_{\mathrm{cs}}^{\mathrm{B}}\right),\left(X^{\mathrm{P}}, \mathrm{r}_{\mathrm{cs}}^{\mathrm{P}}\right)\right] \text { for all } \mathrm{t}=1 \ldots c_{4} \log ^{2} n \\
\mathcal{S}_{\mathrm{t}}^{\mathrm{B}} \subseteq \mathscr{C}_{\mathrm{mcs}}^{\mathrm{pw}}\left[\left(X^{\mathrm{B}}, \mathrm{r}_{\mathrm{cs}}^{\mathrm{B}}\right),\left(X^{\mathrm{P}}, \mathrm{r}_{\mathrm{cs}}^{\mathrm{P}}\right)\right] \text { for all } \mathrm{t}=1 \ldots c_{5}
\end{gathered}
$$

Next, we employ Lemma 11 to establish a lower bound of the throughput of random access on $\mathscr{C}_{\mathrm{mcs}}^{\mathrm{pw}}\left[\left(X^{\mathrm{B}}, \mathrm{r}_{\mathrm{cs}}^{\mathrm{B}}\right),\left(X^{\mathrm{P}}, \mathrm{r}_{\mathrm{cs}}^{\mathrm{P}}\right)\right]$, by the throughput of a corresponding deterministic scheduling scheme as follows:

- For each $\mathcal{S} \in\left\{\mathcal{S}_{\mathrm{t}}^{\mathrm{P}}\right\}_{\mathrm{t}=1}^{c_{4} \log ^{2} n}$, we set $\mathbb{P}^{\text {det }}(\mathcal{S})=\Theta\left(\frac{1}{\log ^{2} n}\right)$

- For each $\mathcal{S} \in\left\{\mathcal{S}_{\mathrm{t}}^{\mathrm{B}}\right\}_{\mathrm{t}=1}^{c_{5}}$, we set $\mathbb{P}^{\operatorname{det}}(\mathcal{S})=\Theta(1)$

- For other $\mathcal{S}$, we equally divide the time among them, such that

$$
\sum_{\mathcal{S} \in \mathscr{C}_{\mathrm{mcs}}^{\mathrm{pw}}\left[\left(X^{\mathrm{B}}, \mathrm{r}_{\mathrm{cs}}^{\mathrm{B}}\right),\left(X^{\mathrm{P}}, \mathrm{r}_{\mathrm{cS}}^{\mathrm{P}}\right)\right] \backslash\left\{\mathcal{S}_{\mathrm{t}}^{\mathrm{P}}\right\}_{\mathrm{t}=1}^{c_{4} \log ^{2} n} \cup\left\{\mathcal{S}_{\mathrm{t}}^{\mathrm{B}}\right\}_{\mathrm{t}=1}^{c_{5}}} \mathbb{P}^{\operatorname{det}}(\mathcal{S})=\Theta(1)
$$

Therefore, this satisfies the sufficient condition in Lemma 11 that $\mathbb{P}^{\text {det }}(\mathcal{S})>0$ for all $\mathcal{S} \in \mathscr{C}_{\mathrm{mcs}}^{\mathrm{pw}}\left[\left(X^{\mathrm{B}}, \mathrm{r}_{\mathrm{cs}}^{\mathrm{B}}\right),\left(X^{\mathrm{P}}, \mathrm{r}_{\mathrm{cs}}^{\mathrm{P}}\right)\right]$. Since such a deterministic scheduling scheme can achieve throughput as $\Omega\left(\frac{1}{\sqrt{n}}\right)$ on a uniform random network w.h.p., it completes the proof by Lemma 11 .

Note that the aggregate model $\mathscr{C}_{\mathrm{mcs}}^{\mathrm{ag}}\left[\left(X^{\mathrm{B}}, \mathrm{r}_{\mathrm{cs}}^{\mathrm{B}}\right),\left(X^{\mathrm{P}}, \mathrm{r}_{\mathrm{cs}}^{\mathrm{P}}\right)\right]$ can be shown in a similar fashion.

\section{B. Dual Channel Dual Carrier-Sensing}

Although the simple dual carrier-sensing decision models d.1)-d.2) can achieve optimal capacity, they suffer from some implementation issues. First, transmitters are required to know the classes of all active links, during the dynamic switching between the dual carrier-sensing operations. In pairwise model $\mathrm{d} .1$ ), this can be achieved by relying on overhearing the physical preambles like MAC addresses of transmitters, and resolving the respective classes. However, this incurs considerably high overhead in dense networks. Particularly, on a uniform random network, the distance for backboneperipheral link is $\Theta(\log n)$. Thus, the induced overhead can scale as large as $\mathrm{O}\left(\log ^{2} n\right)$. Second, in aggregate model d.2), such knowledge of all active links is not available, because a transmitter can only perceive an aggregate power level from all the active links, and is unable to resolve the power levels of individual class.

To address the above implementation issues of dual carriersensing, we are motivated to adopt a system with two frequency channels, in which the communications on the backbone-backbone links are carried out on one frequency channel, while the communications on the peripheral links are carried out on the other channel. That is, the links in class $X^{\mathrm{B}}$ will use one frequency channel with carrier-sensing power threshold as $\mathrm{t}_{\mathrm{cs}}^{\mathrm{B}}$, while the links in class $X^{\mathrm{P}}$ will use the other channel with a threshold $t_{c s}^{P}$. Since conventional CSMA protocols (e.g., IEEE 802.11) often support more than two channels, our scheme can be conveniently implemented on these CSMA protocols.

First, Sec. VII-B1 considers a system that is full-duplex across the two frequency channels. Then, Sec. VII-B2 considers a system that is half-duplex across the two frequency channels that is simpler to implement, but whose conditions for hidden-node free operation are more subtle.

1) Full-Duplexity across Two Frequency Channels: Thus far, we have assumed that the communication on a channel is half-duplex in that when a node transmits, it cannot receive. This is typically the case if one strives for simple transceiver designs. We will continue to assume that a node cannot transmit and receive on the same channel simultaneously. However, we assume full-duplexity across different frequency channels in that simultaneous transmission and reception on different channels are allowed. Specifically, when a node transmits on frequency 1 , it could receive on frequency 2 ; and when a node transmits on frequency 2 , it could receive on frequency 1.

With this set-up, the peripheral nodes will transmit and receive on one of the frequency channels, referred to as the peripheral channel. The backbone nodes will transmit and receive among themselves on the backbone subnet using the other frequency channel, referred to as the backbone channel. 
When transmitting to or receiving from the peripheral nodes, however, the backbones nodes will use the peripheral channel. Thus, a backbone node can conceptually be thought of as consisting of two virtual nodes: a virtual peripheral node for communicating with peripheral nodes associated with it; and a virtual backbone node for relaying packets over the backbone network. This design decouples the operation of the peripheral access subnet from that of the backbone highway.

(I) Carrier-Sensing: The following is the corresponding carrier-sensing decision model:

d.3) Full-duplex dual channel dual aggregate carrier-sensing feasible family: $\mathcal{S} \in \mathscr{D}_{\text {ful }}^{\mathrm{ag}}\left[\left(X^{\mathrm{B}}, \mathrm{t}_{\mathrm{cs}}^{\mathrm{B}}\right),\left(X^{\mathrm{P}}, \mathrm{t}_{\mathrm{cs}}^{\mathrm{P}}\right)\right]$, if and only if for all $i \in \mathcal{S}$,

$$
\mathrm{N}_{0}+\sum_{j \in X^{\mathrm{c}} \cap \mathcal{S}} \mathrm{P}_{\mathrm{tx}}\left|t_{j}-t_{i}\right|^{-\alpha} \leq \mathrm{t}_{\mathrm{cs}}^{\mathrm{c}}
$$

where $i \in X^{\mathrm{c}}$ and $\mathrm{c} \in\{\mathrm{B}, \mathrm{P}\}$.

In essence, a peripheral node will carrier-sense the peripheral channel only. A backbone node will carrier-sense the peripheral channel if it wishes to transmit to a peripheral node, and will carrier-sense the backbone channel if it wishes to transmit to a backbone node.

(II) Throughput: One can easily show that carrier-sensing model d.3) can achieve throughput as $\Omega\left(\frac{1}{\sqrt{n}}\right)$ by relaxing Theorem 3 on two independent frequency channels.

2) Half-Duplexity across Two Frequency Channels: We now consider a system that is half-duplex across the two frequency channels to ease implementation further. A node can still receive on different channels simultaneously. However, we disallow simultaneous transmission and reception, whether on the same channel or different channels. Specifically, we introduce the following constraints:

(i) a node cannot transmit and receive (even on different frequency channels) simultaneously; and

(ii) a node can only transmit on at most one frequency channel at any time.

Constraint $(i)$ is mainly to simplify implementation. When a node transmits, its own transmitted signal power may overwhelm the received signal. Although in principle, the use of a frequency filter may be able to isolate the signals somewhat, the transmit power may be very large compared with the receive power (i.e., extreme near-far problem), such that leakage or crosstalk from the power at the transmit band may not be negligible compared with the receive power. Reference [2] contains a discussion on the need for the assumption of halfduplexity when the transmit and receive frequency channels are the same, but the underlying rationale and principles are the same when the cross-frequency leakage is not negligible.

Constraint (ii) is mainly due to the fact that in ACK-based CSMA schemes (e.g., IEEE 802.11), there is an ACK packet in the reverse direction after the transmission of a DATA packet. If the nodes transmit on two frequency channels and the DATA packets are of different lengths, one of the DATA frames may finish first and the station may end up transmitting DATA and receiving ACK packets at the same time, thus violating constraint $(i)$.
(I) Carrier-Sensing: Let us now consider the implication of constraints ( $i$ ) and (ii) for the carrier sensing operation. The mathematical description of a carrier-sensing decision model that takes care of the constraints is as follows:

d.4) Dual channel dual aggregate carrier-sensing feasible family: $\mathcal{S} \in \mathscr{D}_{\text {haf }}^{\mathrm{ag}}\left[\left(X^{\mathrm{B}}, \mathrm{t}_{\mathrm{cs}}^{\mathrm{B}}\right),\left(X^{\mathrm{P}}, \mathrm{t}_{\mathrm{cs}}^{\mathrm{P}}\right)\right]$, if and only if for all $i \in \mathcal{S}$ and all $\mathrm{c}^{\prime} \in\{\mathrm{B}, \mathrm{P}\}$,

$$
\mathrm{N}_{0}+\sum_{j \in X^{\mathrm{c}^{\prime}} \cap \mathcal{S}} \mathrm{P}_{\mathrm{tx}}\left|t_{j}-t_{i}\right|^{-\alpha} \leq \min \left\{\mathrm{t}_{\mathrm{cs}}^{\mathrm{c}}, \mathrm{t}_{\mathrm{cs}}^{\mathrm{c}^{\prime}}\right\}
$$

where $i \in X^{\mathrm{c}}$ and $\mathrm{c} \in\{\mathrm{B}, \mathrm{P}\}$.

To understand the above, let us first consider the case of a backbone-peripheral link (i.e., $c=P$ ). In this case, either a peripheral node desires to transmit to a backbone node, or a backbone node desires to transmit to a peripheral node. The transmission cannot be allowed if the power sensed is larger than the carrier-sensing threshold of the peripheral channel. It is obvious as to why this should apply to cumulative power sensed on the peripheral channel. But according to the above inequality, the cumulative power sensed on the backbone channel (i.e., for the case where $c^{\prime}=B$ on the left-hand side of the above) should not exceed the peripheral-channel threshold either (Note: on the right-hand side of the above, the threshold of the peripheral channel, $t_{\mathrm{cs}}^{\mathrm{c}}$, is smaller than the threshold of the backbone channel, $\mathrm{t}_{\mathrm{cs}}^{\mathrm{c}^{\prime}}$ ).

The reason for this requirement is constraints $(i)$ and (ii). Consider the following, suppose that a peripheral node wants to transmit to its access backbone node. It is possible that the backbone node is in the midst of a communication with another backbone node. To make sure that the peripheral node does not initiate a transmission to the backbone node in that situation, the peripheral node also has to perform carriersensing on the backbone channel. In the above inequality, to make our analysis simpler and cleaner, we simply set the threshold to that of the peripheral channel. This does not change the order of results. In practice, further implementation optimization is possible (skipped here due to limited space).

Next, we consider the case of a backbone-backbone link (i.e., $c=B$ ). In this case, a backbone node wants to transmit to another backbone node. The transmission is not allowed if the power sensed on the backbone channel is larger than the backbone threshold, or if the power sensed on the peripheral channel is larger than the peripheral threshold. The former is obvious. The latter is due to the fact that the target receiver backbone node may be in the midst of a communication with a peripheral node. Again, further optimization is possible with the latter case. Here, we simply set the threshold to the peripheral threshold, since the order results we want to establish are not compromised.

(II) Throughput: To show that carrier-sensing model d.4) can achieve throughput as $\Omega\left(\frac{1}{\sqrt{n}}\right)$, we first need to properly determine $t_{c s}^{P}$ and $t_{c s}^{B}$. We have to formally show carriersensing decision model d.4) can be implemented practically, by considering dual channel interference models that explicitly incorporate the constraint of half-duplexity across two frequency channels.

e.1) Bi-directional dual channel aggregate SINR feasible 
family: $\mathcal{S} \in \mathscr{B}_{\text {haf }}^{\mathrm{ag}}\left[\left(X^{\mathrm{B}}, \beta^{\mathrm{B}}\right),\left(X^{\mathrm{P}}, \beta^{\mathrm{P}}\right)\right]$, if and only if

1) $\mathcal{S}=\bigcup_{\mathrm{c} \in\{\mathrm{B}, \mathrm{P}\}} \mathcal{S}^{\mathrm{c}}$, where each $\mathcal{S}^{\mathrm{c}} \in \mathscr{B}_{\sin r}^{\mathrm{ag}}\left[X^{\mathrm{c}}, \beta^{\mathrm{c}}\right]$,

2) (half-duplexity constraint) for any pair $i, j \in \mathcal{S}$, $\left\{t_{i}, r_{i}\right\} \cap\left\{t_{j}, r_{j}\right\}=\varnothing$.

Similarly, one can define the respective dual channel interference models for a.0)-a.3),b.0)-b.2).

Theorem 4: There exists a suitable setting of $\left(\mathrm{t}_{\mathrm{cs}}^{\mathrm{B}}, \mathrm{t}_{\mathrm{cs}}^{\mathrm{P}}\right)$, depending on $\left(\beta^{\mathrm{B}}, \beta^{\mathrm{P}}\right)$, such that

$$
\mathscr{B}_{\text {haf }}^{\text {ag }}\left[\left(X^{\mathrm{B}}, \beta^{\mathrm{B}}\right),\left(X^{\mathrm{P}}, \beta^{\mathrm{P}}\right)\right] \supseteq \mathscr{D}_{\text {haf }}^{\mathrm{ag}}\left[\left(X^{\mathrm{B}}, \mathrm{t}_{\mathrm{cs}}^{\mathrm{B}}\right),\left(X^{\mathrm{P}}, \mathrm{t}_{\mathrm{cs}}^{\mathrm{P}}\right)\right]
$$

Theorem 4 establishes a hidden-node-free design for the dual channel dual carrier-sensing decision model. The proof of Theorem 4 is to apply the single-channel hidden-node-free design (Theorem 1) on two independent frequency channels, and then show the half duplexity constraint in e.1) will not affect the setting of hidden-node-free design in d.4).

Lemma 13: For any $c \in\{\mathrm{B}, \mathrm{P}\}$, if

$$
\mathrm{r}_{\mathrm{cs}}^{\mathrm{c}} \geq\left(\frac{1}{\mathrm{P}_{\mathrm{tx}} \mathrm{k}(\alpha)}\left(\mathrm{t}_{\mathrm{cs}}^{\mathrm{c}}-\mathrm{N}_{0}\right)\right)^{-\frac{1}{\alpha}}
$$

then

$$
\mathscr{D}_{\text {haf }}^{\mathrm{ag}}\left[\left(X^{\mathrm{B}}, \mathrm{t}_{\mathrm{cs}}^{\mathrm{B}}\right),\left(X^{\mathrm{P}}, \mathrm{t}_{\mathrm{cs}}^{\mathrm{P}}\right)\right] \supseteq \mathscr{C}_{\mathrm{mcs}}^{\mathrm{pw}}\left[\left(X^{\mathrm{B}}, \mathrm{r}_{\mathrm{cs}}^{\mathrm{B}}\right),\left(X^{\mathrm{P}}, \mathrm{r}_{\mathrm{cs}}^{\mathrm{P}}\right)\right]
$$

Lemma 13 shows that the feasible states of the dual channel dual aggregate carrier-sensing decision model d.4) encompass the feasible states of the pairwise dual carrier carrier-sensing decision model d.1), with suitably chosen carrier-sensing range. Therefore, by Lemma 13 and Theorem 3 , we can show that the implementable dual channel dual aggregate carriersensing decision model d.4) can achieve throughput as as $\Omega\left(\frac{1}{\sqrt{n}}\right)$ on a uniform random network.

Theorem 5: Consider dual channel dual carrier-sensing decision model d.4) on a uniform random network based on backbone-peripheral routing. Using multi-backoff-rate random access, there exists a suitable setting of $\left(t_{\mathrm{cs}}^{\mathrm{B}}, \mathrm{t}_{\mathrm{cs}}^{\mathrm{P}}\right)$, such that

$$
\left.\lambda\left(\mathscr{D}_{\text {haf }}^{\mathrm{ag}}\left[\left(X^{\mathrm{B}}, \mathrm{t}_{\mathrm{cs}}^{\mathrm{B}}\right),\left(X^{\mathrm{P}}, \mathrm{t}_{\mathrm{cs}}^{\mathrm{P}}\right)\right]\right)=\Omega\left(\frac{1}{\sqrt{n}}\right) \quad \text { (w.h.p. }\right)
$$

\section{CONCLUSION}

This paper contains a number of new results and ideas that lend insights and solutions to maximize the achievable capacity in CSMA wireless networks. We formulate a comprehensive set of CSMA models, considering various distributed decision controls and common interference settings from the literature. We establish the relationship between our CSMA models with the existing interference models from the literature. This can characterize both the upper and achievable bounds on the capacity of CSMA networks to be $\Theta\left(\frac{1}{\sqrt{n}}\right)$.

We show that, based on an efficient backbone-peripheral routing scheme and a careful design of dual carrier-sensing and dual channel scheme, hidden-node-free CSMA networks can achieve throughput as $\Omega\left(\frac{1}{\sqrt{n}}\right)$, as optimal as TDMA schemes can on a uniform random network. Along the journey, we also show that normal, single, and homogeneous carrier sensing operation is insufficient to achieve the capacity as optimal as TDMA schemes can on a uniform random network.

\section{REFERENCES}

[1] A. Agarwal and P. R. Kumar. Capacity bounds for ad-hoc and hybrid wireless networks. ACM Computer Communication Review, 34(3):7181, July 2004.

[2] J. G. Andrews, S. Shakkottai, R. Heath, N. Jindal, M. Haenggi, R. Berry, D. Guo, M. Neely, S. Weber, S. Jafar, and A. Yener. Rethinking information theory for mobile ad hoc networks. IEEE Commun. Magazine, 46(12):94-101, December 2008.

[3] F. Baccelli, B. Blaszczyszyn, and P. Muhlethaler. An Aloha protocol for multihop mobile wireless networks. IEEE Trans. Information Theory, 52(2):421-436, February 2006

[4] S. Boyd and L. Vandenberghe. Convex optimization. 2004.

[5] L. Dai and T. T. Lee. Throughput and delay analysis of wireless random access networks. In Proc. CISS 2008, pages 815-820, 2008. The long version available on Arxiv: http://arxiv.org/abs/0805.1300

[6] M. Durvy, O. Dousse, and P. Thiran. Border effects, fairness, and phase transition in large wireless networks. In Proc. IEEE INFOCOM, 2008.

[7] M. Franceschetti, O. Dousse, D. N. C. Tse, and P. Thiran. Closing the gap in the capacity of wireless networks via percolation theory. IEEE Trans. Information Theory, 53(3):1009-1018, March 2007.

[8] L. Fu, S. C. Liew, and J. Huang. Safe carrier sensing range in CSMA network under physical interference model. Technical report, 2009. http://arxiv.org/abs/0901.3611

[9] Y. Gao, D. M. Chiu, and J. C. S. Lui. Determining the end-to-end throughput capacity in multi-hop networks: methodology and applications. In Proc. ACM SIGMETRICS, pages 39-50, 2006.

[10] P. Gupta and P. R. Kumar. The capacity of wireless networks. IEEE Trans. Information Theory, 46(2):388-404, 2000.

[11] L. B. Jiang and S. C. Liew. Improving throughput and fairness by reducing exposed and hidden nodes in 802.11 networks. IEEE Trans. Mobile Computing, 7(1):34-49, January 2008.

[12] L. B. Jiang and J. Walrand. A distributed CSMA algorithm for throughput and utility maximization in wireless networks. In Proc. Allerton Conf. on Comm., Control, and Computing, 2008.

[13] S. Li, Y. Liu, and X.-Y. Li. Capacity of large scale wireless networks under Gaussian channel model. In Proc. ACM MobiCom, 2008.

[14] S. C. Liew, C. Kai, J. Leung, and B. Wong. Back-of-the-envelope computation of throughput distributions in CSMA wireless networks. In Proc. IEEE ICC, 2009.

[15] P. C. Ng and S. C. Liew. Throughput analysis of IEEE802. 11 multi-hop ad hoc networks. IEEE/ACM Trans. Networking, 15(2):309-322, 2007.

[16] K. Xu, M. Gerla, and S. Bae. How effective is the IEEE 802.11 RTS/CTS handshake in ad hoc networks. In Proc. IEEE GLOBECOM, 2002.

[17] F. Xue and P. R. Kumar. Scaling laws for ad hoc wireless networks: An information theoretic approach. Foundations and Trends in Networking, 1(2):145 - 270, July 2006.

\section{APPENDIX}

Lemma 15: For $\gamma>1$, it is straightforward that

$$
\frac{A}{\mathrm{~N}_{0}+\gamma B}<\beta \Rightarrow \frac{A}{\mathrm{~N}_{0}+B}<\gamma \beta
$$

Lemma 16: Let $r_{\mathrm{tx}}=\max _{i \in X}\left|t_{i}-r_{i}\right|$. If there exists $r_{\mathrm{xcl}}>$ $\mathrm{r}_{\mathrm{tx}}$ such that $\left|t_{j}-r_{i}\right| \geq \mathrm{r}_{\mathrm{xcl}}$ for all $i, j \in \mathcal{S}$, then

$$
\sum_{j \in \mathcal{S} \backslash\{i\}}\left|t_{j}-r_{i}\right|^{-\alpha} \leq \mathrm{k}(\alpha)\left(\mathrm{r}_{\mathrm{xcl}}-\mathrm{r}_{\mathrm{tx}}\right)^{-\alpha}
$$

where $\mathrm{k}(\alpha) \triangleq \sum_{k=1}^{\infty} 2\lceil\pi(2 k+2)\rceil k^{-\alpha}$.

Proof: The proof is adopted from [13] (Lemma 3).

For all $i, j \in \mathcal{S}$, we have

$$
\left|t_{i}-t_{j}\right| \geq \mathrm{r}_{\mathrm{xcl}}-\mathrm{r}_{\mathrm{tx}}
$$

For any non-negative integer $k$, let

$T_{k}\left(r_{i}\right)=\left\{j \in X \backslash\{i\}\left|k\left(r_{\mathrm{xcl}}-\mathrm{r}_{\mathrm{tx}}\right) \leq\right| t_{j}-r_{i} \mid<(k+1)\left(\mathrm{r}_{\mathrm{xcl}}-\mathrm{r}_{\mathrm{tx}}\right)\right\}$

By Eq. 41, we obtain

$$
\left|T_{k}\left(r_{i}\right)\right| \leq 2\lceil\pi(2 k+2)\rceil
$$


Hence, it follows that

$$
\begin{aligned}
\sum_{j \in \mathcal{S} \backslash\{i\}}\left|t_{j}-r_{i}\right|^{-\alpha} & \leq \sum_{k=1}^{\infty}\left|T_{k}\left(r_{i}\right)\right|\left(k \cdot\left(\mathrm{r}_{\mathrm{xcl}}-\mathrm{r}_{\mathrm{tx}}\right)\right)^{-\alpha} \\
& \leq \sum_{k=1}^{\infty} 2\lceil\pi(2 k+2)\rceil\left(k \cdot\left(\mathrm{r}_{\mathrm{xcl}}-\mathrm{r}_{\mathrm{tx}}\right)\right)^{-\alpha}
\end{aligned}
$$

Corollary 17: By Lemma 16, if there exists $r_{\mathrm{cs}}$ such that $\left|t_{j}-t_{i}\right| \geq \mathrm{r}_{\mathrm{cs}}$ for all $i, j \in \mathcal{S}$, then

$$
\sum_{j \in \mathcal{S} \backslash\{i\}}\left|t_{j}-t_{i}\right|^{-\alpha} \leq \mathrm{k}(\alpha)\left(\mathrm{r}_{\mathrm{cs}}\right)^{-\alpha}
$$

Lemma 1: If $\Delta \leq \beta^{\frac{1}{\alpha}}-1$, then

$$
\mathscr{U}_{\text {sir }}^{\mathrm{pw}}[X, \Delta] \supseteq \mathscr{U}_{\operatorname{sinr}}^{\mathrm{pw}}[X, \beta] \supseteq \mathscr{U}_{\operatorname{sinr}}^{\mathrm{ag}}[X, \beta]
$$

Proof: $\mathscr{U}_{\mathrm{sinr}}^{\mathrm{pw}}[X, \beta] \supseteq \mathscr{U}_{\mathrm{sinr}}^{\mathrm{ag}}[X, \beta]$ is trivial. $\mathscr{U}_{\text {sir }}^{\mathrm{pw}}[X, \Delta] \supseteq \mathscr{U}_{\mathrm{sinr}}^{\mathrm{pw}}[X, \beta]$ follows from:

$$
\begin{aligned}
\frac{\mathrm{P}_{\mathrm{tx}}\left|t_{i}-r_{i}\right|^{-\alpha}}{\mathrm{N}_{0}+\mathrm{P}_{\mathrm{tx}}\left|t_{j}-r_{i}\right|^{-\alpha} \geq \beta} & \Rightarrow \frac{\mathrm{P}_{\mathrm{tx}}\left|t_{i}-r_{i}\right|^{-\alpha}}{\mathrm{P}_{\mathrm{tx}}\left|t_{j}-r_{i}\right|^{-\alpha}} \geq \beta \\
& \Rightarrow\left|t_{j}-r_{i}\right| \geq \beta^{\frac{1}{\alpha}}\left|t_{i}-r_{i}\right|
\end{aligned}
$$

Lemma 2: Let $r_{\mathrm{tx}}=\max _{i \in X}\left|t_{i}-r_{i}\right|$. If

$$
r_{\mathrm{xcl}} \geq\left(\frac{1}{\mathrm{P}_{\mathrm{tx}} \mathrm{k}(\alpha)}\left(\frac{\mathrm{P}_{\mathrm{tx}}}{\beta} r_{\mathrm{tx}}^{-\alpha}-\mathrm{N}_{0}\right)\right)^{-\frac{1}{\alpha}}+\mathrm{r}_{\mathrm{tx}}
$$

where $\mathrm{k}(\alpha) \triangleq \sum_{k=1}^{\infty} 2\lceil\pi(2 k+2)\rceil k^{-\alpha}$, then

$$
\mathscr{U}_{\mathrm{sinr}}^{\mathrm{ag}}[X, \beta] \supseteq \mathscr{U}_{\mathrm{fr}}^{\mathrm{pw}}\left[X, \mathrm{r}_{\mathrm{xcl}}, \mathrm{r}_{\mathrm{tx}}\right]
$$

Proof: Suppose $\mathcal{S} \in \mathscr{U}_{\mathrm{fr}}^{\mathrm{pw}}\left[X, \mathrm{r}_{\mathrm{xcl}}, \mathrm{r}_{\mathrm{t} \times}\right]$ and $i \in \mathcal{S}$. By Lemma 16, we obtain:

$$
\frac{\mathrm{P}_{\mathrm{tx}}\left|t_{i}-r_{i}\right|^{-\alpha}}{\mathrm{N}_{0}+\sum_{j \in \mathcal{S} \backslash\{i\}} \mathrm{P}_{\mathrm{tx}}\left|t_{j}-r_{i}\right|^{-\alpha}} \geq \frac{\mathrm{P}_{\mathrm{tx}} \mathrm{r}_{\mathrm{tx}}^{-\alpha}}{\mathrm{N}_{0}+\mathrm{P}_{\mathrm{tx}} \mathrm{k}(\alpha)\left(\mathrm{r}_{\mathrm{xcl}}-\mathrm{r}_{\mathrm{tx}}\right)^{-\alpha}}
$$

Hence,

$$
\begin{aligned}
& r_{\mathrm{xcl}} \geq\left(\frac{1}{P_{\mathrm{tx}} k(\alpha)}\left(\frac{P_{\mathrm{tx}}}{\beta} r_{\mathrm{tx}}^{-\alpha}-\mathrm{N}_{0}\right)\right)^{-\frac{1}{\alpha}}+r_{\mathrm{tx}} \\
& \Leftrightarrow \frac{\mathrm{P}_{\mathrm{tx}} r_{\mathrm{t}}^{-\alpha}}{\mathrm{N}_{0}+\mathrm{P}_{\mathrm{tx}} \mathrm{k}(\alpha)\left(\mathrm{r}_{\mathrm{xcl}}-r_{\mathrm{tx}}\right)^{-\alpha}} \geq \beta \Rightarrow \mathcal{S} \in \mathscr{U}_{\mathrm{sinr}}^{\mathrm{ag}}[X, \beta]
\end{aligned}
$$

Lemma 3: If $r_{x \mathrm{xl}}^{\prime} \geq r_{\mathrm{xcl}}+2 r_{\mathrm{tx}}$, then

$$
\mathscr{U}_{\mathrm{fr}}^{\mathrm{pw}}\left[X, \mathrm{r}_{\mathrm{xcl}}, \mathrm{r}_{\mathrm{tx}}\right] \supseteq \mathscr{B}_{\mathrm{fr}}^{\mathrm{pw}}\left[X, \mathrm{r}_{\mathrm{xcl}}, \mathrm{r}_{\mathrm{tx}}\right] \supseteq \mathscr{U}_{\mathrm{fr}}^{\mathrm{pw}}\left[X, \mathrm{r}_{\mathrm{xcl}}^{\prime}, \mathrm{r}_{\mathrm{tx}}\right]
$$

Proof: By Lemma 4 and set $\Delta=\mathrm{r}_{\mathrm{xcl}} / \mathrm{r}_{\mathrm{tx}}-1$ and $\Delta^{\prime}=$ $\mathrm{r}_{\mathrm{xcl}}^{\prime} / \mathrm{r}_{\mathrm{tx}}-1$

Lemma 4 can be proven by applying Lemma 5 and letting $\mathrm{N}_{0}=0, \Delta=\beta^{\frac{1}{\alpha}}-1$ and $\Delta^{\prime}=\beta^{\prime \frac{1}{\alpha}}-1$. For clarity of presentation, we present the proof of Lemma 4 in order to reveal the basic idea of Lemma 5 in the simpler context when $\mathrm{N}_{0}=0$.

Lemma 4: If $\Delta^{\prime} \geq \Delta+2$, then

$$
\mathscr{U}_{\text {sir }}^{\mathrm{pw}}[X, \Delta] \supseteq \mathscr{B}_{\text {sir }}^{\mathrm{pw}}[X, \Delta] \supseteq \mathscr{U}_{\mathrm{sir}}^{\mathrm{pw}}\left[X, \Delta^{\prime}\right]
$$

Proof: Follows from Lemma 6

Lemma 5: If $\beta^{\prime} \geq\left(2+\beta^{\frac{1}{\alpha}}\right)^{\alpha}$, then

$$
\mathscr{U}_{\operatorname{sinr}}^{\mathrm{pw}}[X, \beta] \supseteq \mathscr{B}_{\operatorname{sinr}}^{\mathrm{pw}}[X, \beta] \supseteq \mathscr{U}_{\operatorname{sinr}}^{\mathrm{pw}}\left[X, \beta^{\prime}\right]
$$

Proof: This is proven in a similar fashion as Lemma 4 Suppose $\mathcal{S} \in \mathscr{B}_{\operatorname{sinr}}^{\mathrm{pw}}[X, \beta]$. First, note that $\mathrm{P}_{\mathrm{tx}}\left|t_{i}-r_{i}\right|^{-\alpha} \geq$ $\beta \mathrm{N}_{0}$ for all $i \in X$. Otherwise, $t_{i}$ will be unable to transmit to $r_{i}$ even without interference. Second,

$$
\begin{gathered}
\frac{\mathrm{P}_{\mathrm{tx}}\left|t_{i}-r_{i}\right|^{-\alpha}}{\mathrm{N}_{0}+\mathrm{P}_{\mathrm{tx}}(\operatorname{dist}(i, j))^{-\alpha}}<\beta \\
\Rightarrow\left(\frac{1}{\mathrm{P}_{\mathrm{tx}}}\left(\frac{\mathrm{P}_{\mathrm{tx}}}{\beta}\left|t_{i}-r_{i}\right|^{-\alpha}-\mathrm{N}_{0}\right)\right)^{-\frac{1}{\alpha}}>\operatorname{dist}(i, j)
\end{gathered}
$$

The last inequality is due to the fact that $-\frac{1}{\alpha}<0$ and $\mathrm{P}_{\mathrm{tx}} \mid t_{i}-$ $\left.r_{i}\right|^{-\alpha} \geq \beta \mathrm{N}_{0}$.

We need to show that

$$
\mathcal{S} \cup\{i\} \notin \mathscr{B}_{\mathrm{sinr}}^{\mathrm{pw}}[X, \beta] \Rightarrow \mathcal{S} \cup\{i\} \notin \mathscr{U}_{\mathrm{sinr}}^{\mathrm{pw}}\left[X,\left(2+\beta^{\frac{1}{\alpha}}\right)^{\alpha}\right]
$$

Suppose $\mathcal{S} \cup\{i\} \notin \mathscr{B}_{\mathrm{sinr}}^{\mathrm{pw}}[X, \beta]$ for some given link $i$. Then there are four cases as follows.

1): Suppose $\frac{P_{\mathrm{tx}}\left|t_{i}-r_{i}\right|^{-\alpha}}{\mathrm{N}_{0}+\mathrm{P}_{\mathrm{tx}}\left|t_{j}-r_{i}\right|^{-\alpha}}<\beta$ for some $j \in \mathcal{S}$. This is trivial that $\mathcal{S} \cup\{i\} \notin \mathscr{U}_{\mathrm{sinr}}^{\mathrm{pw}}[X, \beta]$.

2): Suppose for some $j \in \mathcal{S}$ that

$$
\frac{\mathrm{P}_{\mathrm{tx}}\left|t_{i}-r_{i}\right|^{-\alpha}}{\mathrm{N}_{0}+\mathrm{P}_{\mathrm{tx}}\left|r_{j}-r_{i}\right|^{-\alpha}}<\beta
$$

Without loss of generality, we also assume

$$
\left|t_{i}-r_{i}\right| \geq\left|t_{j}-r_{j}\right|
$$

Otherwise, if $\left|t_{j}-r_{j}\right| \geq\left|t_{i}-r_{i}\right|$, then

$$
\begin{aligned}
\left|r_{j}-r_{i}\right| & <\left(\frac{1}{\mathrm{P}_{\mathrm{tx}}}\left(\frac{\mathrm{P}_{\mathrm{tx}}}{\beta}\left|t_{i}-r_{i}\right|^{-\alpha}-\mathrm{N}_{0}\right)\right)^{-\frac{1}{\alpha}} \\
& \leq\left(\frac{1}{\mathrm{P}_{\mathrm{tx}}}\left(\frac{\mathrm{P}_{\mathrm{tx}}}{\beta}\left|t_{j}-r_{j}\right|^{-\alpha}-\mathrm{N}_{0}\right)\right)^{-\frac{1}{\alpha}}
\end{aligned}
$$

Therefore, we can equivalently assume

$$
\frac{\mathrm{P}_{\mathrm{tx}}\left|t_{j}-r_{j}\right|^{-\alpha}}{\mathrm{N}_{0}+\mathrm{P}_{\mathrm{tx}}\left|r_{j}-r_{i}\right|^{-\alpha}}<\beta \text { and }\left|t_{j}-r_{j}\right| \geq\left|t_{i}-r_{i}\right| \text {, }
$$

by inter-changing $i$ and $j$.

Next, we obtain:

$$
\begin{aligned}
\left|t_{j}-r_{i}\right| & \leq\left|r_{j}-r_{i}\right|+\left|t_{j}-r_{j}\right| \\
& <\left(\frac{1}{\mathrm{P}_{\mathrm{tx}}}\left(\frac{\mathrm{P}_{\mathrm{tx}}}{\beta}\left|t_{i}-r_{i}\right|^{-\alpha}-\mathrm{N}_{0}\right)\right)^{-\frac{1}{\alpha}}+\left|t_{j}-r_{j}\right| \quad \text { by Eqns. (57), (55) } \\
& \leq\left(\frac{1}{\mathrm{P}_{\mathrm{tx}}}\left(\frac{\mathrm{P}_{\mathrm{tx}}}{\beta}\left|t_{i}-r_{i}\right|^{-\alpha}-\mathrm{N}_{0}\right)\right)^{-\frac{1}{\alpha}}+\left|t_{i}-r_{i}\right| \quad \text { by Eqn. (62) } \\
& \leq\left(1+\beta^{-\frac{1}{\alpha}}\right)\left(\frac{1}{\mathrm{P}_{\mathrm{tx}}}\left(\frac{\mathrm{P}_{\mathrm{tx}}}{\beta}\left|t_{i}-r_{i}\right|^{-\alpha}-\mathrm{N}_{0}\right)\right)^{-\frac{1}{\alpha}}
\end{aligned}
$$

The last inequality is due to the fact that when $N_{0} \geq 0$,

$$
\frac{\beta}{\mathrm{P}_{\mathrm{tx}}}\left(\frac{\mathrm{P}_{\mathrm{tx}}}{\beta}\left|t_{i}-r_{i}\right|^{-\alpha}-\mathrm{N}_{0}\right) \geq\left|t_{i}-r_{i}\right|^{-\alpha}
$$

By Lemma 15 setting $\gamma=\left(1+\beta^{-\frac{1}{\alpha}}\right)^{\alpha}$, we obtain:

$$
\frac{\mathrm{P}_{\mathrm{tx}}\left|t_{i}-r_{i}\right|^{-\alpha}}{\mathrm{N}_{0}+\mathrm{P}_{\mathrm{t} \times}\left|t_{j}-r_{i}\right|^{-\alpha}}<\left(1+\beta^{-\frac{1}{\alpha}}\right)^{\alpha} \beta=\left(1+\beta^{\frac{1}{\alpha}}\right)^{\alpha}
$$

3): Suppose $\frac{P_{\mathrm{tx}}\left|t_{i}-r_{i}\right|^{-\alpha}}{\mathrm{N}_{0}+\mathrm{P}_{\mathrm{t} \mathrm{x}}\left|t_{j}-t_{i}\right|^{-\alpha}}<\beta$ for some $j \in \mathcal{S}$. This is shown in a similar way as Case 2.

4): Suppose for some $j \in \mathcal{S}$ that

$$
\frac{\mathrm{P}_{\mathrm{tx}}\left|t_{i}-r_{i}\right|^{-\alpha}}{\mathrm{N}_{0}+\mathrm{P}_{\mathrm{tx}}\left|t_{i}-r_{j}\right|^{-\alpha}}<\beta
$$


In addition, we assume

$$
\frac{\mathrm{P}_{\mathrm{tx}}\left|t_{j}-r_{j}\right|^{-\alpha}}{\mathrm{N}_{0}+\mathrm{P}_{\mathrm{tx}}\left|t_{i}-r_{j}\right|^{-\alpha}} \geq \beta
$$

Otherwise, it reduces to Case 1 by inter-changing $i$ and j. Hence, we obtain:

$$
\begin{aligned}
& \left(\frac{1}{\mathrm{P}_{\mathrm{tx}}}\left(\frac{\mathrm{P}_{\mathrm{tx}}}{\beta}\left|t_{j}-r_{j}\right|^{-\alpha}-\mathrm{N}_{0}\right)\right)^{-\frac{1}{\alpha}} \\
\leq & \left|t_{i}-t_{j}\right|<\left(\frac{1}{\mathrm{P}_{\mathrm{tx}}}\left(\frac{\mathrm{P}_{\mathrm{tx}}}{\beta}\left|t_{i}-r_{i}\right|^{-\alpha}-\mathrm{N}_{0}\right)\right)^{-\frac{1}{\alpha}} \\
\Rightarrow & \left|t_{j}-r_{j}\right|<\left|t_{i}-r_{i}\right|
\end{aligned}
$$

Next, we obtain:

$$
\left|t_{j}-r_{i}\right|
$$$$
\leq\left|t_{j}-r_{j}\right|+\left|r_{j}-t_{i}\right|+\left|t_{i}-r_{i}\right|
$$

$<\left|t_{j}-r_{j}\right|+\left(\frac{1}{\mathrm{P}_{\mathrm{tx}}}\left(\frac{\mathrm{P}_{\mathrm{tx}}}{\beta}\left|t_{j}-r_{j}\right|^{-\alpha}-\mathrm{N}_{0}\right)\right)^{-\frac{1}{\alpha}}+\left|t_{i}-r_{i}\right|$ by Eqn. 65

$$
<\left(1+2 \beta^{-\frac{1}{\alpha}}\right)\left(\frac{1}{\mathrm{P}_{\mathrm{tx}}}\left(\frac{\mathrm{P}_{\mathrm{tx}}}{\beta}\left|t_{i}-r_{i}\right|^{-\alpha}-\mathrm{N}_{0}\right)\right)^{-\frac{1}{\alpha}}
$$

By Lemma 15, setting $\gamma=\left(2+\beta^{-\frac{1}{\alpha}}\right)^{\alpha}$, we obtain:

$$
\frac{\mathrm{P}_{\mathrm{tx}}\left|t_{i}-r_{i}\right|^{-\alpha}}{\mathrm{N}_{0}+\mathrm{P}_{\mathrm{tx}}\left|t_{j}-r_{i}\right|^{-\alpha}}<\beta\left(1+2 \beta^{-\frac{1}{\alpha}}\right)^{\alpha}=\left(2+\beta^{\frac{1}{\alpha}}\right)^{\alpha}
$$

Therefore, $\mathcal{S} \cup\{i\} \notin \mathscr{U}_{\operatorname{sinr}}^{\mathrm{pw}}\left[X, \beta^{\prime}\right]$. This proves Eqn. (56).

Lemma 6: If $\beta^{\prime} \geq\left(2+\beta^{\frac{1}{\alpha}}\right)^{\alpha}$, then

$$
\mathscr{U}_{\text {sinr }}^{\mathrm{ag}}[X, \beta] \supseteq \mathscr{B}_{\operatorname{sinr}}^{\mathrm{ag}}[X, \beta] \supseteq \mathscr{U}_{\text {sinr }}^{\mathrm{ag}}\left[X, \beta^{\prime}\right]
$$

Proof: Suppose $\mathcal{S} \in \mathscr{B}_{\operatorname{sinr}}^{\text {ag }}[X, \beta]$. We need to show

$$
\mathcal{S} \cup\{i\} \notin \mathscr{B}_{\operatorname{sinr}}^{\mathrm{ag}}[X, \beta] \Rightarrow \mathcal{S} \cup\{i\} \notin \mathscr{U}_{\operatorname{sinr}}^{\mathrm{ag}}\left[X,\left(2+\beta^{\frac{1}{\alpha}}\right)^{\alpha}\right]
$$

First, we assume

$$
\begin{aligned}
& \frac{\mathrm{P}_{\mathrm{tx}}\left|t_{j}-r_{j}\right|^{-\alpha}}{\mathrm{N}_{0}+\mathrm{P}_{\mathrm{tx}}(\operatorname{dist}(j, i))^{-\alpha}} \geq \beta \\
\Rightarrow & \frac{\mathrm{P}_{\mathrm{tx}}\left|t_{j}-r_{j}\right|^{-\alpha}}{\mathrm{P}_{\mathrm{tx}}(\operatorname{dist}(j, i))^{-\alpha}} \geq \beta \Rightarrow\left|t_{j}-r_{j}\right| \leq \beta^{-\frac{1}{\alpha}}(\operatorname{dist}(j, i))
\end{aligned}
$$

and

$$
\begin{aligned}
& \frac{\mathrm{P}_{\mathrm{tx}}(\operatorname{dist}(j, i))^{-\alpha}}{\mathrm{N}_{0}+\mathrm{P}_{\mathrm{tx}}(\operatorname{dist}(i, j))^{-\alpha}} \geq \beta \\
\Rightarrow & \frac{\mathrm{P}_{\mathrm{tx}}\left|t_{i}-r_{i}\right|^{-\alpha}}{\mathrm{P}_{\mathrm{tx}}(\operatorname{dist}(i, j))^{-\alpha}} \geq \beta \Rightarrow\left|t_{i}-r_{i}\right| \leq \beta^{-\frac{1}{\alpha}}(\operatorname{dist}(i, j))
\end{aligned}
$$

Otherwise, we complete the proof by Lemma 5 such that

$$
\mathcal{S} \cup\{i\} \notin \mathscr{U}_{\operatorname{sinr}}^{\mathrm{ag}}\left[X,\left(2+\beta^{\frac{1}{\alpha}}\right)^{\alpha}\right]
$$

Next, we obtain:

$$
\begin{aligned}
\left|t_{j}-r_{i}\right| & \leq\left|t_{j}-r_{j}\right|+\left|r_{j}-r_{i}\right| \\
& \leq \beta^{-\frac{1}{\alpha}}\left|r_{j}-r_{i}\right|+\left|r_{j}-r_{i}\right| \\
\Rightarrow\left|t_{j}-r_{i}\right| & \leq\left(1+\beta^{-\frac{1}{\alpha}}\right)\left|r_{j}-r_{i}\right| \\
\left|t_{j}-r_{i}\right| & \leq\left|t_{i}-r_{i}\right|+\left|t_{j}-t_{i}\right| \\
\Rightarrow\left|t_{j}-r_{i}\right| & \leq\left(1+\beta^{-\frac{1}{\alpha}}\right)\left|t_{j}-t_{i}\right| \\
\left|t_{j}-r_{i}\right| & \leq\left|t_{j}-r_{j}\right|+\left|r_{j}-t_{i}\right|+\left|t_{i}-r_{i}\right| \\
\Rightarrow\left|t_{j}-r_{i}\right| & \leq\left(1+2 \beta^{-\frac{1}{\alpha}}\right)\left|r_{j}-t_{i}\right|
\end{aligned}
$$$$
\leq \beta^{-\frac{1}{\alpha}}\left|r_{j}-r_{i}\right|+\left|r_{j}-r_{i}\right| \quad \text { by Eqn. (72) }
$$

by Eqn. (72)

by Eqn. (71)
Therefore,

$$
\left|t_{j}-r_{i}\right|<\left(1+2 \beta^{-\frac{1}{\alpha}}\right) \cdot \operatorname{dist}(i, j)
$$

Also, since $\mathcal{S} \cup\{i\} \notin \mathscr{B}_{\text {sinr }}^{\text {ag }}[X, \beta]$, we obtain:

$$
\begin{aligned}
& \frac{\mathrm{P}_{\mathrm{tx}}\left|t_{i}-r_{i}\right|^{-\alpha}}{\mathrm{N}_{0}+\sum_{j \in \mathcal{S} \backslash\{i\}} \mathrm{P}_{\mathrm{tx}}(\operatorname{dist}(i, j))^{-\alpha}}<\beta \\
\Rightarrow & \sum_{j \in \mathcal{S} \backslash\{i\}}(\operatorname{dist}(i, j))^{-\alpha}>\frac{1}{\mathrm{P}_{\mathrm{tx}}}\left(\frac{\mathrm{P}_{\mathrm{tx}}}{\beta}\left|t_{i}-r_{i}\right|^{-\alpha}-\mathrm{N}_{0}\right) \\
\Rightarrow & \sum_{j \in \mathcal{S} \backslash\{i\}}\left(1+2 \beta^{-\frac{1}{\alpha}}\right)^{\alpha}\left|t_{j}-r_{i}\right|^{-\alpha}>\frac{1}{\mathrm{P}_{\mathrm{tx}}}\left(\frac{\mathrm{P}_{\mathrm{tx}}}{\beta}\left|t_{i}-r_{i}\right|^{-\alpha}-\mathrm{N}_{0}\right) \text { by Eqn. 75) }
\end{aligned}
$$

By Lemma 15, setting $\gamma=\left(2+\beta^{-\frac{1}{\alpha}}\right)^{\alpha}$, we obtain:

$$
\frac{\mathrm{P}_{\mathrm{tx}}\left|t_{i}-r_{i}\right|^{-\alpha}}{\mathrm{N}_{0}+\sum_{j \neq i} \mathrm{P}_{\mathrm{tx}}\left|t_{j}-r_{i}\right|^{\alpha}}<\beta\left(1+2 \beta^{-\frac{1}{\alpha}}\right)^{\alpha}=\left(2+\beta^{\frac{1}{\alpha}}\right)^{\alpha}
$$

Finally, we complete the proof by combining with Lemma 5 .

Lemma 7: If $\mathrm{r}_{\mathrm{tx}}=\max _{i \in X}\left|t_{i}-r_{i}\right|$ and $\mathrm{r}_{\mathrm{cs}} \geq \mathrm{r}_{\mathrm{xcl}}+2 \mathrm{r}_{\mathrm{tx}}$,

$$
\mathscr{C}^{\mathrm{pw}}\left[X, \mathrm{r}_{\mathrm{xcl}}\right] \supseteq \mathscr{B}_{\mathrm{fr}}^{\mathrm{pw}}\left[X, \mathrm{r}_{\mathrm{xcl}}, \mathrm{r}_{\mathrm{tx}}\right] \supseteq \mathscr{C}^{\mathrm{pw}}\left[X, \mathrm{r}_{\mathrm{cs}}\right]
$$

Proof: It can be proven in a similar fashion as Lemma 3 , where we replace constraint $\left|t_{j}-r_{i}\right| \geq \mathrm{r}_{\mathrm{xcl}}$ by $\left|t_{j}-t_{i}\right| \geq \mathrm{r}_{\mathrm{cs}}$.

Lemma 8: If $\mathrm{t}_{\mathrm{cs}} \leq \mathrm{N}_{0}+\mathrm{P}_{\mathrm{tx}} \mathrm{r}_{\mathrm{cs}}^{-\alpha}$, then

$$
\mathscr{C}^{\mathrm{pw}}\left[X, \mathrm{r}_{\mathrm{cs}}\right] \supseteq \mathscr{C}^{\mathrm{ag}}\left[X, \mathrm{t}_{\mathrm{cs}}\right]
$$

Proof: The proof is straightforward.

Lemma 9: If $\mathrm{r}_{\mathrm{cs}} \geq\left(\frac{1}{\mathrm{P}_{\mathrm{t} \times} \mathrm{k}(\alpha)}\left(\mathrm{t}_{\mathrm{cs}}-\mathrm{N}_{0}\right)\right)^{-\frac{1}{\alpha}}$, then

$$
\mathscr{C}^{\mathrm{ag}}\left[X, \mathrm{t}_{\mathrm{cs}}\right] \supseteq \mathscr{C}^{\mathrm{pw}}\left[X, \mathrm{r}_{\mathrm{cs}}\right]
$$

Proof: Suppose $\mathcal{S} \in \mathscr{C}^{\mathrm{pw}}\left[X, \mathrm{r}_{\mathrm{cs}}\right], i \in \mathcal{S}$ and $i \in X$. By Corollary 17

$$
\sum_{j \in \mathcal{S} \backslash\{i\}} \mathrm{P}_{\mathrm{tx}}\left|t_{j}-t_{i}\right|^{-\alpha} \leq \mathrm{P}_{\mathrm{tx}} \mathrm{k}(\alpha)\left(\mathrm{r}_{\mathrm{cs}}\right)^{-\alpha}
$$

Next, we obtain:

$$
\begin{aligned}
& \mathrm{r}_{\mathrm{cs}} \geq\left(\frac{1}{\mathrm{P}_{\mathrm{txk}} \mathrm{k}(\alpha)}\left(\mathrm{t}_{\mathrm{cs}}-\mathrm{N}_{0}\right)\right)^{-\frac{1}{\alpha}} \\
\Rightarrow & \mathrm{N}_{0}+\mathrm{P}_{\mathrm{tx}} \mathrm{k}(\alpha)\left(\mathrm{r}_{\mathrm{cs}}\right)^{-\alpha} \leq \mathrm{t}_{\mathrm{cs}} \\
\Rightarrow & \mathrm{N}_{0}+\sum_{j \in \mathcal{S} \backslash\{i\}} \mathrm{P}_{\mathrm{tx}}\left|t_{j}-t_{i}\right|^{-\alpha} \leq \mathrm{t}_{\mathrm{cs}}
\end{aligned}
$$

Hence, $\mathcal{S} \in \mathscr{C}^{\mathrm{ag}}\left[X, \mathrm{t}_{\mathrm{cs}}\right]$.

Lemma 10: $\left\langle\mathscr{F}_{\mathrm{cs}}[X], \nu\right\rangle$ is a reversible Markov process. The stationary distribution for each $\mathcal{S} \in \mathscr{F}_{\mathrm{cs}}[X]$ is:

$$
\mathbb{P}_{\nu}(\mathcal{S})=\frac{\exp \left(\sum_{i \in \mathcal{S}} \log \nu_{i}\right)}{\sum_{\mathcal{S}^{\prime} \in \mathscr{F}_{\mathrm{cs}}[X]} \exp \left(\sum_{j \in \mathcal{S}^{\prime}} \log \nu_{j}\right)}
$$

Proof: Eqn. (83) satisfies the detailed balanced eqn: $\exp \left(\sum_{j \in\{i\} \cup \mathcal{S}} \log \nu_{j}\right)=\exp \left(\sum_{j \in \mathcal{S}} \log \nu_{j}\right) \cdot \exp \left(\log \nu_{i}\right)$

$$
\Rightarrow \mathbb{P}_{\nu}(\{i\} \cup \mathcal{S})=\mathbb{P}_{\nu}(\mathcal{S}) \cdot \nu_{i}
$$

Hence, $\left\langle\mathscr{F}_{\mathrm{cs}}[X], \nu\right\rangle$ is a reversible Markov process, Eqn. 83 is the stationary distribution.

Lemma 11: Given a deterministic scheduling scheme by Eqns. (71), (72) $\left(\mathcal{S}_{\mathrm{t}}\right)_{\mathrm{t}=1}^{\mathrm{m}}$, let the fraction of time spent in $\mathcal{S} \in \mathscr{F}_{\mathrm{cs}}[X]$ be (74) $\mathbb{P}^{\text {det }}(\mathcal{S})=\frac{1}{\mathrm{~m}} \sum_{\mathrm{t}=1}^{\mathrm{m}} \mathbb{1}\left(\mathcal{S}=\mathcal{S}_{\mathrm{t}}\right)$. If $\mathbb{P}^{\mathrm{det}}(\mathcal{S})>0$ for all 
$\mathcal{S} \in \mathscr{F}_{\mathrm{cs}}[X]$, then there exists count-down rate $\nu$, such that for each transmitter-receiver pair $i \in X$, it satisfies:

$$
\mathfrak{c}_{i}^{\operatorname{det}}\left[\left(\mathcal{S}_{\mathrm{t}}\right)_{\mathrm{t}=1}^{\mathrm{n}}\right] \leq \mathfrak{c}_{i}^{\text {rand }}\left[\left\langle\mathscr{F}_{\mathrm{cs}}[X], \nu\right\rangle\right]
$$

Proof: Let $\mathfrak{c}_{i}^{\text {det }} \triangleq \mathfrak{c}_{i}^{\operatorname{det}}\left[\left(\mathcal{S}_{\mathrm{t}}\right)_{\mathrm{t}=1}^{\mathrm{n}}\right]$, and $\mathfrak{c}_{i}^{\text {rand }}(\nu) \triangleq$ $\mathfrak{c}_{i}^{\text {rand }}\left[\left\langle\mathscr{F}_{\mathrm{cs}}[X], \nu\right\rangle\right]$. We start by considering the following feasibility problem:

$$
\text { P1: } \begin{array}{rl}
\max _{\mathbf{z} \geq 0} & 0 \\
\text { subject to } & \sum_{\mathcal{S} \in \mathscr{F}_{\mathrm{cs}}[X]: i \in \mathcal{S}} z_{\mathcal{S}} \geq \mathfrak{c}_{i}^{\text {det }}, \text { for all } i \in X \\
\sum_{\mathcal{S} \in \mathscr{F}_{\mathrm{cs}}[X]} z_{\mathcal{S}}=1
\end{array}
$$

Its corresponding Lagranian problem is given by:

$$
\begin{aligned}
& \operatorname{minmax}_{\lambda \geq 0 \mathbf{z} \geq 0}-\sum_{i \in X} \lambda_{i} \mathfrak{c}_{i}^{\text {det }}+\sum_{\mathcal{S} \in \mathscr{F}_{\mathrm{cs}}[X]} z_{\mathcal{S}} \sum_{i \in \mathcal{S}} \lambda_{i} \\
& \text { subject to } \sum_{\mathcal{S} \in \mathscr{F}_{\mathrm{cs}}[X]} z_{\mathcal{S}}=1
\end{aligned}
$$

The subproblem in $\mathbf{z}$ is the Maximum Weighted Independent Set (MWIS) problem, which is a combinatorial problem, whose optimal value is given on the left hand side of the following formula and is approximated by the log-sum-exp function on the right hand side [4]:

$$
\max _{\mathcal{S} \in \mathscr{F}_{\mathrm{cs}}[X]} \sum_{i \in \mathcal{S}} \lambda_{i} \approx \log \left(\sum_{\mathcal{S} \in \mathscr{F}_{\mathrm{cs}}[X]} \exp \left(\sum_{i \in \mathcal{S}} \lambda_{i}\right)\right) .
$$

Define the log-sum-exp function as $g(\boldsymbol{\lambda})$. It is known that ts conjugate function is a convex function and is given by [4]

$$
g^{*}(\boldsymbol{z})= \begin{cases}\sum_{\mathcal{S} \in \mathscr{F}_{\mathrm{cS}}[X]} z_{\mathcal{S}} \log z_{\mathcal{S}} & \text { if } \boldsymbol{z} \geq 0 \text { and } \mathbf{1}^{T} \boldsymbol{z}=1 \\ \infty & \text { otherwise }\end{cases}
$$

Further, $g(\boldsymbol{\lambda})$ is convex and closed; hence, the conjugate of its conjugate $g^{*}(\boldsymbol{z})$ is itself. Specifically, $g(\boldsymbol{\lambda})$ is the optimal value of a concave optimization problem:

$$
\begin{aligned}
g(\boldsymbol{\lambda})= & \max _{\mathbf{z} \geq 0} \sum_{\mathcal{S} \in \mathscr{F}_{\mathrm{cs}}[X]} z_{\mathcal{S}} \sum_{i \in \mathcal{S}} \lambda_{i}-\sum_{\mathcal{S} \in \mathscr{F}_{\mathrm{cs}}[X]} z_{\mathcal{S}} \log \left(z_{\mathcal{S}}(87)\right. \\
& \text { subject to } \sum_{\mathcal{S} \in \mathscr{F}_{\mathrm{cs}}[X]} z_{\mathcal{S}}=1
\end{aligned}
$$

Therefore, by the log-sum-exp approximation in Eq. 85, we implicitly solve an approximated version of the MWIS problem, off by an entropy term $-\sum_{\mathcal{S} \in \mathscr{F}_{\mathrm{cs}}[X]} z_{\mathcal{S}} \log \left(z_{\mathcal{S}}\right)$. By solving the Karush-Kuhn-Tucker (KKT) conditions [4], we obtain the optimal solution to the approximated problem in Eq. 87) as follows,

$$
z_{\mathcal{S}}(\boldsymbol{\lambda})=\frac{\exp \left(\sum_{i \in \mathcal{S}} \lambda_{i}\right)}{\sum_{\mathcal{S}^{\prime} \in \mathscr{F}_{\mathrm{cs}}[X]} \exp \left(\sum_{j \in \mathcal{S}^{\prime}} \lambda_{j}\right)} .
$$

Comparing Eq. (83) and (88), we observe that $z_{\mathcal{S}}(\boldsymbol{\lambda})$ is the percentage of time state $\mathcal{S}$ being activated under CSMA scheduling, with every link $i \in X$ counts down with a rate of $\nu_{i}=\exp \left(\lambda_{i}\right)$.

After the log-sum-exp approximation in Eq. (85), the Lagranian problem becomes

$$
\begin{array}{ll}
\operatorname{minmax}_{\lambda \geq 0 \mathbf{z}>0} & -\sum_{i \in X} \lambda_{i} \mathfrak{c}_{i}^{\text {det }}+\sum_{\mathcal{S} \in \mathscr{F}_{\mathrm{cs}}[X]} z_{\mathcal{S}} \sum_{i \in \mathcal{S}} \lambda_{i} \\
& -\sum_{\mathcal{S} \in \mathscr{F}_{\mathrm{cs}}[X]} z_{\mathcal{S}} \log \left(z_{\mathcal{S}}\right) \\
\text { subject to } & \sum_{\mathcal{S} \in \mathscr{F}_{\mathrm{cs}}[X]} z_{\mathcal{S}}=1
\end{array}
$$

We reverse-engineer to get the corresponding primal problem as the following entropy maximization problem:

$$
\begin{aligned}
& \text { P2: } \quad \max _{\mathbf{z} \geq 0}-\sum_{\mathcal{S} \in \mathscr{F}_{\mathrm{cS}}[X]} z_{\mathcal{S}} \log \left(z_{\mathcal{S}}\right) \\
& \text { subject to } \sum_{\mathcal{S} \in \mathscr{F}_{\mathrm{cs}}[X]: i \in \mathcal{S}} z_{\mathcal{S}} \geq \mathfrak{c}_{i}^{\text {det }} \text {, for all } i \in X \\
& \sum_{\mathcal{S} \in \mathscr{F}_{\mathrm{cs}}[X]} z_{\mathcal{S}}=1
\end{aligned}
$$

By the setting in the lemma statement, the linear constraint set of problem $\mathbf{P 2}$ contains at least one relaitve interior point, and thus problem P2 satisfies Slater's condition [4]. Consequently, the optimal dual and primal solutions to its Lagranian problem in Eq. (89) $\boldsymbol{\lambda}^{*}$ and $\boldsymbol{z}_{\mathcal{S}}\left(\boldsymbol{\lambda}^{*}\right)$ exist, and $\boldsymbol{z}_{\mathcal{S}}\left(\boldsymbol{\lambda}^{*}\right)$ is the optimal solution to problem $\mathbf{P 2}$ and is feasible. In other words, by CSMA scheduling with every link $i \in X$ counts down with a rate of $\nu_{i}=\exp \left(\lambda_{i}^{*}\right)$, we obtain that for every link $i \in X$ its link throughput satisfies:

$$
\mathfrak{c}_{i}^{\text {rand }}(\nu)=\sum_{\mathcal{S} \in \mathscr{F}_{\mathrm{cs}}[X]: i \in \mathcal{S}} \boldsymbol{z}_{\mathcal{S}}\left(\boldsymbol{\lambda}^{*}\right) \geq \mathfrak{c}_{i}^{\text {det }} .
$$

This completes the proof.

Theorem 4: There exists a suitable setting of $\left(\mathrm{t}_{\mathrm{cs}}^{\mathrm{B}}, \mathrm{t}_{\mathrm{cs}}^{\mathrm{P}}\right)$, depending on $\left(\beta^{\mathrm{B}}, \beta^{\mathrm{P}}\right)$, such that

$$
\mathscr{B}_{\text {haf }}^{\text {ag }}\left[\left(X^{\mathrm{B}}, \beta^{\mathrm{B}}\right),\left(X^{\mathrm{P}}, \beta^{\mathrm{P}}\right)\right] \supseteq \mathscr{D}_{\text {haf }}^{\mathrm{ag}}\left[\left(X^{\mathrm{B}}, \mathrm{t}_{\mathrm{cs}}^{\mathrm{B}}\right),\left(X^{\mathrm{P}}, \mathrm{t}_{\mathrm{cs}}^{\mathrm{P}}\right)\right]
$$

Proof: First, we note that

$$
\begin{aligned}
& \mathscr{B}_{\text {haf }}^{\mathrm{ag}}\left[\left(X^{\mathrm{B}}, \beta^{\mathrm{B}}\right),\left(X^{\mathrm{P}}, \beta^{\mathrm{P}}\right)\right] \\
= & \left\{\bigcup_{\mathrm{c} \in\{\mathrm{B}, \mathrm{P}\}} \mathcal{S}^{\mathrm{c}} \mid \mathcal{S}^{\mathrm{c}} \in \mathscr{B}_{\operatorname{sinr}}^{\mathrm{ag}}\left[X^{\mathrm{c}}, \beta^{\mathrm{c}}\right]\right\} \\
& \bigcup\left\{\mathcal{S} \mid i, j \in \mathcal{S},\left\{t_{i}, r_{i}\right\} \cap\left\{t_{j}, r_{j}\right\}=\varnothing\right\}
\end{aligned}
$$

By Theorem 1, for each $c \in\{B, P\}$, there exists a suitable $\mathrm{t}_{\mathrm{cs}}^{\mathrm{c}}$, depending on $\beta^{\mathrm{c}}$, such that

$$
\mathscr{B}_{\operatorname{sinr}}^{\mathrm{ag}}\left[X^{\mathrm{c}}, \beta^{\mathrm{c}}\right] \supseteq \mathscr{C}^{\mathrm{ag}}\left[X^{\mathrm{c}}, \mathrm{t}_{\mathrm{cs}}^{\mathrm{c}}\right]
$$

Then, suppose $\mathcal{S} \in \mathscr{D}_{\text {haf }}^{\mathrm{ag}}\left[\left(X^{\mathrm{B}}, \mathrm{t}_{\mathrm{cs}}^{\mathrm{B}}\right),\left(X^{\mathrm{P}}, \mathrm{t}_{\mathrm{cs}}^{\mathrm{P}}\right)\right], i \in \mathcal{S}$ and $i \in X^{\mathrm{c}}$ for some $\mathrm{c} \in\{\mathrm{B}, \mathrm{P}\}$. We obtain:

$$
\begin{aligned}
& \mathrm{N}_{0}+\sum_{j \in X^{\mathrm{c}^{\prime}} \cap \mathcal{S}} \mathrm{P}_{\mathrm{tx}}\left|t_{j}-t_{i}\right|^{-\alpha} \leq \min \left\{\mathrm{t}_{\mathrm{cs}}^{\mathrm{c}}, \mathrm{t}_{\mathrm{cs}}^{\mathrm{c}^{\prime}}\right\} \text { for all } \mathrm{c}^{\prime} \in\{\mathrm{B}, \mathrm{P}\} \\
\Rightarrow & \mathrm{N}_{0}+\sum_{j \in X^{\mathrm{c}} \cap \mathcal{S}} \mathrm{P}_{\mathrm{tx}}\left|t_{j}-t_{i}\right|^{-\alpha} \leq \mathrm{t}_{\mathrm{cs}}^{\mathrm{c}}
\end{aligned}
$$

Hence, this follows that

$$
\begin{aligned}
\left\{\bigcup_{\mathrm{c} \in\{\mathrm{B}, \mathrm{P}\}}\right. & \left.\mathcal{S}^{\mathrm{c}} \mid \mathcal{S}^{\mathrm{c}} \in \mathscr{C}^{\mathrm{ag}}\left[X^{\mathrm{c}}, \mathrm{t}_{\mathrm{cs}}^{\mathrm{c}}\right]\right\} \\
& \supseteq \mathscr{D}_{\text {haf }}^{\mathrm{ag}}\left[\left(X^{\mathrm{B}}, \mathrm{t}_{\mathrm{cs}}^{\mathrm{B}}\right),\left(X^{\mathrm{P}}, \mathrm{t}_{\mathrm{cs}}^{\mathrm{P}}\right)\right]
\end{aligned}
$$

Next, we need to show there exists a suitable $t_{c s}^{c}$, such that

$$
\begin{aligned}
\{\mathcal{S} \mid i, j \in \mathcal{S} & \left.,\left\{t_{i}, r_{i}\right\} \cap\left\{t_{j}, r_{j}\right\}=\varnothing\right\} \\
& \supseteq \mathscr{D}_{\text {haf }}^{\mathrm{ag}}\left[\left(X^{\mathrm{B}}, \mathrm{t}_{\mathrm{cs}}^{\mathrm{B}}\right),\left(X^{\mathrm{P}}, \mathrm{t}_{\mathrm{cs}}^{\mathrm{P}}\right)\right]
\end{aligned}
$$


If $i \in X^{\mathrm{c}}$ and $j \in X^{\mathrm{c}}$, then Eqn. (96) follows from the tree diagram Fig. 4 that there exists a suitable $t_{c s}^{c}$, such that

$$
\mathscr{B}_{\text {sir }}^{\mathrm{pw}}\left[X^{\mathrm{c}}, \Delta^{\mathrm{c}}\right] \supseteq \mathscr{C}^{\mathrm{ag}}\left[X^{\mathrm{c}}, \mathrm{t}_{\mathrm{cs}}^{\mathrm{c}}\right]
$$

for any $\Delta^{c}>0$. Otherwise, without loss of generality, we consider $i \in X^{\mathrm{c}}$ and $j \in X^{\mathrm{c}^{\prime}}$, and $\mathrm{t}_{\mathrm{cs}}^{\mathrm{c}}<\mathrm{t}_{\mathrm{cs}}^{\mathrm{c}^{\prime}}$. Then, Eqn. (96) follows from that there exists a suitable $\mathrm{t}_{\mathrm{cS}}^{\mathrm{c}}$, such that

$$
\mathscr{B}_{\text {sir }}^{\mathrm{pw}}\left[X^{\mathrm{c}} \cup\{j\}, \Delta^{\mathrm{c}}\right] \supseteq \mathscr{C}^{\mathrm{ag}}\left[X^{\mathrm{c}} \cup\{j\}, \mathrm{t}_{\mathrm{cs}}^{\mathrm{c}}\right] \supseteq \mathscr{C}^{\mathrm{ag}}\left[X^{\mathrm{c}}, \mathrm{t}_{\mathrm{cs}}^{\mathrm{c}}\right]
$$

for any $\Delta^{\mathrm{c}}>0$. Taking the maximum $\mathrm{t}_{\mathrm{cs}}^{\mathrm{c}}$ in Eqns. (92), (95), (96), we then complete the proof.

Lemma 13: For any $\mathrm{c} \in\{\mathrm{B}, \mathrm{P}\}$, if

$$
\mathrm{r}_{\mathrm{cs}}^{\mathrm{c}} \geq\left(\frac{1}{\mathrm{P}_{\mathrm{t} \times} \mathrm{k}(\alpha)}\left(\mathrm{t}_{\mathrm{cs}}^{\mathrm{c}}-\mathrm{N}_{0}\right)\right)^{-\frac{1}{\alpha}},
$$

then

$$
\mathscr{D}_{\text {haf }}^{\mathrm{ag}}\left[\left(X^{\mathrm{B}}, \mathrm{t}_{\mathrm{cs}}^{\mathrm{B}}\right),\left(X^{\mathrm{P}}, \mathrm{t}_{\mathrm{cs}}^{\mathrm{P}}\right)\right] \supseteq \mathscr{C}_{\mathrm{mcs}}^{\mathrm{pw}}\left[\left(X^{\mathrm{B}}, \mathrm{r}_{\mathrm{cs}}^{\mathrm{B}}\right),\left(X^{\mathrm{P}}, \mathrm{r}_{\mathrm{cs}}^{\mathrm{P}}\right)\right]
$$

Proof: Suppose $\mathcal{S} \in \mathscr{C}_{\mathrm{mcs}}^{\mathrm{pw}}\left[\left(X^{\mathrm{B}}, \mathrm{r}_{\mathrm{cs}}^{\mathrm{B}}\right),\left(X^{\mathrm{P}}, \mathrm{r}_{\mathrm{cs}}^{\mathrm{P}}\right)\right], i \in \mathcal{S}$ and $i \in X^{\mathrm{c}}$. For each $\mathrm{c}^{\prime} \in\{\mathrm{B}, \mathrm{P}\}$, by Corollary 17 ,

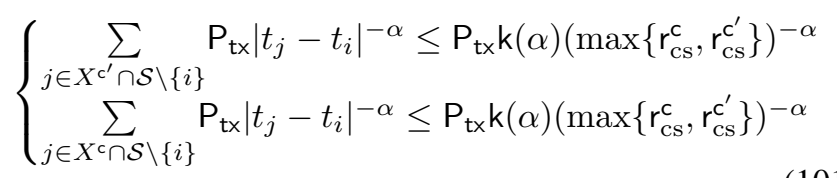

Next, we obtain:

$$
\begin{aligned}
& \mathrm{r}_{\mathrm{cs}}^{\mathrm{c}^{\prime}} \geq\left(\frac{1}{\mathrm{P}_{\mathrm{tx}} \mathrm{k}(\alpha)}\left(\mathrm{t}_{\mathrm{cS}}^{\mathrm{c}^{\prime}}-\mathrm{N}_{0}\right)\right)^{-\frac{1}{\alpha}} \\
\Rightarrow & \max \left\{\mathrm{r}_{\mathrm{cs}}^{\mathrm{c}}, \mathrm{r}_{\mathrm{cs}}^{\mathrm{r}^{\prime}}\right\} \geq\left(\frac{1}{\mathrm{P}_{\mathrm{tx}} \mathrm{k}(\alpha)}\left(\mathrm{t}_{\mathrm{cs}}^{\mathrm{c}^{\prime}}-\mathrm{N}_{0}\right)\right)^{-\frac{1}{\alpha}} \\
\Rightarrow & \mathrm{N}_{0}+\mathrm{P}_{\mathrm{t} \mathrm{t} \mathrm{k}} \mathrm{k}(\alpha)\left(\max \left\{\mathrm{r}_{\mathrm{cs}}^{\mathrm{c}}, \mathrm{r}_{\mathrm{cs}}^{c^{\prime}}\right\}\right)^{-\alpha} \leq \mathrm{t}_{\mathrm{cs}}^{\mathrm{c}^{\prime}}
\end{aligned}
$$

By Eqns. 101, (102), it follows that

$$
\begin{aligned}
& \mathrm{N}_{0}+\sum_{j \in X^{\mathrm{c}^{\prime}} \cap \mathcal{S} \backslash\{i\}} \mathrm{P}_{\mathrm{tx}}\left|t_{j}-t_{i}\right|^{-\alpha} \leq \mathrm{t}_{\mathrm{cs}}^{\mathrm{c}^{\prime}} \\
& \mathrm{N}_{0}+\sum_{j \in X^{\mathrm{c}} \cap \mathcal{S} \backslash\{i\}} \mathrm{P}_{\mathrm{tx}}\left|t_{j}-t_{i}\right|^{-\alpha} \leq \mathrm{t}_{\mathrm{cs}}^{\mathrm{c}^{\prime}}
\end{aligned}
$$

Inter-changing $c$ and $c^{\prime}$ in Eqn. (104), we obtain:

$$
\mathrm{N}_{0}+\sum_{j \in X^{c^{\prime}} \cap \mathcal{S} \backslash\{i\}} \mathrm{P}_{\mathrm{tx} \times}\left|t_{j}-t_{i}\right|^{-\alpha} \leq \mathrm{t}_{\mathrm{cs}}^{\mathrm{c}}
$$

Hence, by Eqns. (103), (105), we obtain $\mathcal{S} \in$ $\mathscr{D}_{\text {haf }}^{\mathrm{ag}}\left[\left(X^{\mathrm{B}}, \mathrm{t}_{\mathrm{cs}}^{\mathrm{B}}\right),\left(X^{\mathrm{P}}, \mathrm{t}_{\mathrm{cs}}^{\mathrm{P}}\right)\right]$.
Lemma 18: Let $\mathrm{r}_{\mathrm{tx}}=\max _{i \in X}\left|t_{i}-r_{i}\right|$. If

$$
r_{\mathrm{xcl}} \geq\left(\frac{1}{\mathrm{P}_{\mathrm{tx}}}\left(\frac{\mathrm{P}_{\mathrm{tx}}}{\beta} \mathrm{r}_{\mathrm{tx}}^{-\alpha}-\mathrm{N}_{0}\right)\right)^{-\frac{1}{\alpha}}
$$

then

$$
\mathscr{U}_{\mathrm{sinr}}^{\mathrm{pw}}[X, \beta] \supseteq \mathscr{U}_{\mathrm{fr}}^{\mathrm{pw}}\left[X, \mathrm{r}_{\mathrm{xcl}}, \mathrm{r}_{\mathrm{tx}}\right]
$$

Proof: Suppose $\mathcal{S} \in \mathscr{U}_{\mathrm{fr}}^{\mathrm{pw}}\left[X, \mathrm{r}_{\mathrm{xcl}}, \mathrm{r}_{\mathrm{tx}}\right]$ and $i, j \in \mathcal{S}$. Then,

$$
\begin{aligned}
& r_{\mathrm{xcl}} \geq\left(\frac{1}{\mathrm{P}_{\mathrm{tx}}}\left(\frac{\mathrm{P}_{\mathrm{tx}}}{\beta} \mathrm{r}_{\mathrm{tx}}^{-\alpha}-\mathrm{N}_{0}\right)\right)^{-\frac{1}{\alpha}} \\
& \Leftrightarrow \frac{\mathrm{P}_{\mathrm{tx}} \mathrm{r}_{\mathrm{tx}}^{-\alpha}}{\mathrm{N}_{\mathrm{t}}+\mathrm{P}_{\mathrm{tx}} \mathrm{r}_{\mathrm{xcl}}^{-\alpha}} \geq \beta \Leftrightarrow \frac{\mathrm{P}_{\mathrm{t}}\left|t_{i}-r_{i}\right|^{-\alpha}}{\mathrm{N}_{0}+\mathrm{P}_{\mathrm{tx}}\left|t_{j}-r_{i}\right|^{-\alpha}} \geq \beta
\end{aligned}
$$

for all $i, j \in \mathcal{S}$. Hence, $\mathcal{S} \in \mathscr{U}_{\operatorname{sinr}}^{\mathrm{ag}}[X, \beta]$.

Lemma 19: Let $\mathrm{r}_{\mathrm{tx}}=\max _{i \in X}\left|t_{i}-r_{i}\right|$. If

$$
r_{\mathrm{cs}} \geq\left(\frac{1}{\mathrm{P}_{\mathrm{tx}}}\left(\frac{\mathrm{P}_{\mathrm{tx}}}{\beta} r_{\mathrm{tx}}^{-\alpha}-\mathrm{N}_{0}\right)\right)^{-\frac{1}{\alpha}}+2 r_{\mathrm{tx}}
$$

then

$$
\mathscr{U}_{\mathrm{sinr}}^{\mathrm{pw}}[X, \beta] \supseteq \mathscr{C}^{\mathrm{pw}}\left[X, \mathrm{r}_{\mathrm{cs}}\right]
$$

Proof: Follows from Lemma 18, 5, 7 